This item was submitted to Loughborough's Research Repository by the author.

Items in Figshare are protected by copyright, with all rights reserved, unless otherwise indicated.

\title{
Polymorphisms and adiabatic chaos
}

PLEASE CITE THE PUBLISHED VERSION

http://dx.doi.org/10.1017/S0143385709001060

PUBLISHER

(c) Cambridge University Press

VERSION

VoR (Version of Record)

LICENCE

CC BY-NC-ND 4.0

REPOSITORY RECORD

Neishtadt, Anatoly, and Dmitry Treschev. 2019. "Polymorphisms and Adiabatic Chaos". figshare. https://hdl.handle.net/2134/15560. 
This item was submitted to Loughborough's Institutional Repository (https://dspace.lboro.ac.uk/) by the author and is made available under the following Creative Commons Licence conditions.

\section{creative
commons}

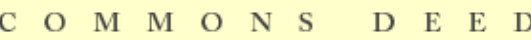

Attribution-NonCommercial-NoDerivs 2.5

You are free:

- to copy, distribute, display, and perform the work

Under the following conditions:

Attribution. You must attribute the work in the manner specified b the author or licensor.

Noncommercial. You may not use this work for commercial purposes.

No Derivative Works. You may not alter, transform, or build upon this work.

- For any reuse or distribution, you must make clear to others the license terms of this work.

- Any of these conditions can be waived if you get permission from the copyright holder.

Your fair use and other rights are in no way affected by the above.

This is a human-readable summary of the Leqal Code (the full license).

\section{Disclaimer 만}

For the full text of this licence, please go to: http://creativecommons.org/licenses/by-nc-nd/2.5/ 


\title{
Polymorphisms and adiabatic chaos
}

\author{
A. NEISHTADT $\dagger \ddagger$ and D. TRESCHEV $\S$ \\ $\dagger$ Department of Mathematical Sciences, Loughborough University, Loughborough, \\ LE11 3TU, UK \\ † Space Research Institute, Profsoyuznaya 84/32, Moscow, 117997, Russia \\ $\S$ Steklov Mathematical Institute, Gubkina 8, Moscow, 119991, Russia
}

(Received 14 August 2008 and accepted in revised form 28 August 2009)

\begin{abstract}
At the end of the last century Vershik introduced some dynamical systems, called polymorphisms. Systems of this kind are multivalued self-maps of an interval, where (roughly speaking) each branch has some probability. By definition, the standard Lebesgue measure should be invariant. Unexpectedly, some class of polymorphisms appeared in the problem of destruction of an adiabatic invariant after a multiple passage through a separatrix. We discuss ergodic properties of polymorphisms from this class.
\end{abstract}

\section{Introduction}

In [7] and [8] Vershik introduced some dynamical systems called polymorphisms. Any polymorphism is a dynamical system determined by a multivalued self-map of a measure space $(M, \mu)$. We will consider the case $\dagger$ when $M$ is the interval $[0,1]$ and $\mu$ is the standard Lebesgue measure $d x$.

A polymorphism $T$ is defined as the ordered diagram

$$
\left([0,1]_{x}, d x\right) \stackrel{\pi_{x}}{\longleftarrow}\left([0,1]_{x} \times[0,1]_{y}, v\right) \stackrel{\pi_{y}}{\longrightarrow}\left([0,1]_{y}, d y\right),
$$

where $\pi_{x}$ and $\pi_{y}$ are projections to the $x$-component and $y$-components respectively of the product $[0,1]_{x} \times[0,1]_{y}$ and $v$ is a probability measure such that

$$
\pi_{x} v=d x \text { and } \pi_{y} v=d y .
$$

The dynamical interpretation of $T$ is as follows: $T$ maps randomly any measurable set $\Lambda \subset[0,1]_{x}$ to the interval $[0,1]_{y}$ so that the probability of a measurable set $\Omega \subset[0,1]_{y}$ equals $v(\Lambda \times \Omega)$.

Unexpectedly, polymorphisms appeared in the problem of destruction of an adiabatic invariant after a multiple passage through a separatrix. However the measures $v$, appearing

$\dagger$ Recall that, if $(M, \mu)$ is a Lebesgue space, where $\mu$ is a measure without atoms, it is metrically isomorphic to $([0,1], d x)$. 

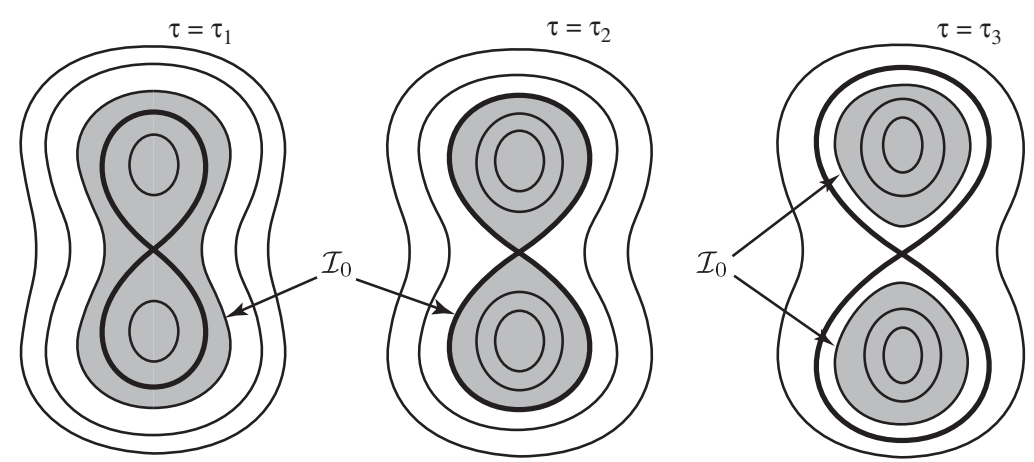

FIGURE 1. Phase portraits of the 'frozen' system. Grey domains correspond to the same value $\mathcal{I}_{0}$ of the adiabatic invariant.

in this way, have a special structure: they are supported on several curves, lying in $[0,1]_{x} \times[0,1]_{y}$. Below we define this class of polymorphisms and discuss ergodic properties of some its representatives.

Our plan is as follows. In $\S 2$ we explain how polymorphisms appear in the problem of adiabatic chaos. This section is independent of the remaining part of the paper and a reader, not interested in motivations, in principle can skip it. In $\S 3$ we define the class of polymorphisms which is the subject of this paper and present basic constructions including the Perron-Frobenius operator, the concepts of ergodicity and mixing. In $\$ 4$ we define some important subclass of polymorphisms and present several existence statements for this class. In $\$ 5$ we present examples of polymorphisms with non-ergodic dynamics. In $\S 6$ we discuss metric properties of the Perron-Frobenius operator for polymorphisms from $\S 4$. As a corollary we obtain two theorems which present sufficient conditions for mixing. In $\$ 7$ we study ergodic properties of a two-parametric family of piecewise-linear polymorphisms $T_{\beta, s}$. Appendices contain proofs of some technical statements.

\section{Motivation}

Consider the Hamiltonian system with one and a half degrees of freedom

$$
\dot{x}=\partial H / \partial y, \quad \dot{y}=-\partial H / \partial x, \quad H=H(x, y, \varepsilon t),
$$

where dots are derivatives with respect to time $t, H$ is $\hat{\tau}$-periodic in $\tau=\varepsilon t$,

$$
H(x, y, \tau)=H(x, y, \tau+\hat{\tau}),
$$

and $\varepsilon$ is a small parameter.

First, consider the case of fixed ('frozen') $\tau$. Then we have a system with one degree of freedom (the 'frozen system'). Suppose that for any fixed $\tau$ the level lines of $H$ (the phase portrait of the frozen system) are as in Figure 1. More precisely, we assume that for any $\tau$ there is a hyperbolic fixed point $\hat{z}(\tau)$ with two homoclinic separatrix loops ('upper' $\gamma_{+}(\tau)$ and 'lower' $\gamma_{-}(\tau)$ ), and the picture depends smoothly on $\tau$.

For small $\varepsilon \neq 0$ the system has an adiabatic invariant: the action $\mathcal{I}$ in the 'frozen system'. The separatrices divide the phase space $\{x, y\}$ of the 'frozen system' into three 
domains: $D_{+}(\tau), D_{-}(\tau)$, and $D_{0}(\tau)$ with the boundaries

$$
\partial D_{+}=\gamma_{+} \cup \hat{z}, \quad \partial D_{-}=\gamma_{-} \cup \hat{z}, \quad \partial D_{0}=\gamma_{+} \cup \gamma_{-} \cup \hat{z} .
$$

In each of these domains the action $\mathcal{I}=\mathcal{I}(H, \tau)$ equals the area inside the curve $H=$ constant, see [1]. It is smooth outside the separatrix and discontinuous on the separatrix. For a motion far from the separatrices the value of $\mathcal{I}$ for a moving phase point remains constant with accuracy of order $\varepsilon$ on time intervals of order $1 / \varepsilon$. Approximation in which the value of $\mathcal{I}$ is considered as a constant in the process of the motion is called the adiabatic approximation.

For the case when a phase point crosses the separatrices the adiabatic approximation should be modified as follows. The value of $\mathcal{I}$ is considered as a constant up to an arrival of the phase point at a separatrix. At this time moment the phase point can change the domain. Different phase points which arrive at the separatrix simultaneously can be captured into different domains. A certain probability can be assigned to the capture into each domain (see below). After the capture into a certain domain the value of the action is considered as a constant up to the next arrival at the separatrix. Estimates of the accuracy of this approximation can be found in [4]. This form of the adiabatic approximation allows us to describe the dynamics as an evolution of a set of 'adiabatic' phase points. Each of the phase points in this set has a value of $\mathcal{I}$ calculated by means of the adiabatic approximation and a certain probability assigned to it.

Now we define a multivalued map $\mathcal{I} \mapsto T(\mathcal{I})$ which associates with $\mathcal{I}$ at the time moment $\tau=0$ its value $T(\mathcal{I})$ at the time moment $\tau=\hat{\tau}$ in the adiabatic approximation.

First, we determine the domain, where the map $T$ is defined. Let

$$
\begin{gathered}
A_{+}(\tau)=\int_{\gamma_{+}(\tau)} y d x, \quad A_{-}(\tau)=\int_{\gamma_{-}(\tau)} y d x, \\
A_{0}(\tau)=A_{+}(\tau)+A_{-}(\tau)
\end{gathered}
$$

be the areas bounded by the upper, lower separatrix loops and of all the separatrix respectively. These quantities can be regarded as areas of the domains $D_{+}, D_{-}$, and the complement of $D_{0}$ respectively.

Let $U(\tau)$ be the disjoint union of three intervals

$$
U_{ \pm}(\tau)=\left(0, A_{ \pm}(\tau)\right), \quad U_{0}(\tau)=\left(A_{0}(\tau), C\right),
$$

where $C=\max _{\tau} A_{0}(\tau)$. We consider $U(\tau)$ together with the standard Lebesgue measure $d u$. By (2.1) the total measure

$$
|U(\tau)|=A_{+}(\tau)+A_{-}(\tau)+C-A_{0}(\tau)=C
$$

does not depend on $\tau$. Here we assume that if $\mathcal{I} \in U_{+}(\tau)\left(\mathcal{I} \in U_{-}(\tau), \mathcal{I} \in U_{0}(\tau)\right)$, the corresponding phase point lies in $D_{+}(\tau)\left(D_{-}(\tau), D_{0}(\tau)\right)$.

The domain of $T$ is $U(0)$. For an arbitrary partition

$$
0=\tau_{0}<\tau_{1}<\cdots<\tau_{N}=\hat{\tau}
$$

the map $T$ is the composition

$$
T=T_{\tau_{N-1}}^{\tau_{N}} \circ \cdots \circ T_{\tau_{1}}^{\tau_{2}} \circ T_{\tau_{0}}^{\tau_{1}}
$$


where $T_{\tau_{s}}^{\tau_{s+1}}: U\left(\tau_{s}\right) \rightarrow U\left(\tau_{s+1}\right)$ associates with $\mathcal{I}=\left.\mathcal{I}\right|_{\tau=\tau_{s}}$ the quantity $\left.\mathcal{I}\right|_{\tau=\tau_{s+1}}=$ $T_{\tau_{s}}^{\tau_{s+1}}(\mathcal{I})$. To define this map, we will have to consider several cases.

(a) Suppose that $\mathcal{I} \in U_{*}\left(\tau_{s}\right), * \in\{+,-, 0\}$, and the equation

$$
A_{*}(\tau)=\mathcal{I}, \quad \tau \in\left[\tau_{s}, \tau_{s+1}\right]
$$

has no solution. Then $T_{\tau_{s}}^{\tau_{s+1}}(\mathcal{I})=\mathcal{I}$. This corresponds to the fact that $\mathcal{I}$ is preserved if the phase point does not cross the separatrix.

In other cases the phase points cross the separatrix and we will assume that the interval $\left[\tau_{s}, \tau_{s+1}\right]$ is so small that this event takes place only once on $\left[\tau_{s}, \tau_{s+1}\right]$. Moreover, for convenience we assume that this happens at some time moment $\tilde{\tau} \in\left(\tau_{s}, \tau_{s+1}\right)$.

$\left(\mathrm{b}_{0}\right)$ Suppose that $\mathcal{I} \in U_{0}\left(\tau_{s}\right)$ and

$$
A_{0}(\tilde{\tau})=\mathcal{I} \quad \text { for some } \tilde{\tau} \in\left(\tau_{s}, \tau_{s+1}\right)
$$

This means that the area $A_{0}(\tau)$ increases and for $\tau=\tilde{\tau}$ becomes equal to $\mathcal{I}$. In particular, $A_{0}^{\prime}(\tilde{\tau}) \geq 0$. (Here and below 'prime' denotes derivative with respect to $\tau$.) For $\tau>\tilde{\tau}$ the phase points fall into the domains $D_{+}$and $D_{-}$:

$$
T_{\tau_{s}}^{\tau_{s+1}}(\mathcal{I})= \begin{cases}A_{+}(\tilde{\tau}) \in U_{+}\left(\tau_{s+1}\right) & \text { with probability } p_{0}^{+}(\tilde{\tau}) \\ A_{-}(\tilde{\tau}) \in U_{-}\left(\tau_{s+1}\right) & \text { with probability } p_{0}^{-}(\tilde{\tau})\end{cases}
$$

where

$$
p_{0}^{+}(\tilde{\tau})=\frac{A_{+}^{\prime}(\tilde{\tau})}{A_{0}^{\prime}(\tilde{\tau})}, \quad p_{0}^{-}(\tilde{\tau})=\frac{A_{-}^{\prime}(\tilde{\tau})}{A_{0}^{\prime}(\tilde{\tau})} \text { provided } A_{ \pm}^{\prime}(\tilde{\tau}) \geq 0 .
$$

If $A_{+}^{\prime}(\tilde{\tau})$ or $A_{-}^{\prime}(\tilde{\tau})$ is negative, the phase points fall into the domain bounded by the increasing loop with probability 1 .

$\left(\mathrm{b}_{+}\right)$Suppose that $\mathcal{I} \in U_{+}\left(\tau_{s}\right)$ and

$$
A_{+}(\tilde{\tau})=\mathcal{I} \quad \text { for some } \tilde{\tau} \in\left(\tau_{s}, \tau_{s+1}\right) .
$$

This means that the area $A_{+}(\tau)$ decreases and for $\tau=\tilde{\tau}$ becomes equal to $\mathcal{I}$. In particular, $A_{+}^{\prime}(\tilde{\tau}) \leq 0$. The phase points leave $D_{+}$and for $\tau>\tilde{\tau}$ are captured into $D_{0}$ and $D_{-}$:

$$
T_{\tau_{s}}^{\tau_{s+1}}(\mathcal{I})= \begin{cases}A_{0}(\tilde{\tau}) \in U_{0}\left(\tau_{s+1}\right) & \text { with probability } p_{+}^{0}(\tilde{\tau}) \\ A_{-}(\tilde{\tau}) \in U_{-}\left(\tau_{s+1}\right) & \text { with probability } p_{+}^{-}(\tilde{\tau})\end{cases}
$$

where

$$
p_{+}^{0}(\tilde{\tau})=\frac{-A_{0}^{\prime}(\tilde{\tau})}{-A_{+}^{\prime}(\tilde{\tau})}, \quad p_{+}^{-}(\tilde{\tau})=\frac{A_{-}^{\prime}(\tilde{\tau})}{-A_{+}^{\prime}(\tilde{\tau})} \operatorname{provided} A_{0}^{\prime}(\tilde{\tau})<0 \text { and } A_{-}^{\prime}(\tilde{\tau})>0 .
$$

If $A_{0}^{\prime}(\tilde{\tau})>0$, the phase points fall into $D_{-}$, and if $A_{-}^{\prime}(\tilde{\tau})<0$, into $D_{0}$ with probability 1 .

(b-) The case $\mathcal{I} \in U_{-}\left(\tau_{s}\right)$ is analogous to $\left(\mathrm{b}_{+}\right)$.

Obviously, $T$, defined by (2.3), does not depend on partition (2.2).

Dynamical systems, generated by these multivalued maps are expected to have generically strong ergodic properties which implies a fast stochastization in the original Hamiltonian system if $\mathcal{I}_{0} \in U(0)$.

\section{CAMBRIDGE JDURNALS}


Remark 2.1. If $A_{+}(\tau) \equiv A_{-}(\tau)$, the map $T$ is, in a sense, trivial:

$$
T(\mathcal{I})= \begin{cases}\mathcal{I} \in U_{0}(0) & \text { if } \mathcal{I} \in U_{0}(0), \\ \mathcal{I} \in U_{+}(0) \text { with probability } 1 / 2 & \text { if } \mathcal{I} \in U_{ \pm}(0), \\ \mathcal{I} \in U_{-}(0) \text { with probability } 1 / 2 & \text { if } \mathcal{I} \in U_{ \pm}(0) .\end{cases}
$$

Chaos in this situation is produced by a more delicate effect: small error terms appeared because of scattering on the separatrix (recall that these terms are skipped in the adiabatic approximation we use here) $[\mathbf{2}, \mathbf{5}, \mathbf{6}]$.

The multi-valued map $T$ can be regarded as a collection of maps $\varphi_{j}: I_{j} \rightarrow U(0)$, $j=1, \ldots, J$, where $I_{j} \subset U(0)$ are intervals. Any point $u \in U(0)$ lying in $I_{s_{1}}, \ldots, I_{s_{k}}$ has the images $\varphi_{s_{1}}(u), \ldots, \varphi_{s_{k}}(u)$ with probabilities $p_{s_{1}}(u), \ldots, p_{s_{k}}(u)$ respectively. Without loss of generality we can assume that $\varphi_{j}$ are monotone because in case of necessity we can divide $I_{j}$ by subintervals of monotonicity of the $\varphi_{j}$.

THEOREM 1. The map $T$ preserves the measure $d u$ on $U(0)$ : for almost all $u \in U(0)$

$$
\sum_{l=1}^{k} \frac{p_{s_{l}}\left(v_{l}\right)}{\left|\varphi_{s_{l}}^{\prime}\left(v_{l}\right)\right|}=1,
$$

where $v_{1}, \ldots, v_{k}$ are all preimages of $u: \varphi_{s_{l}}\left(v_{l}\right)=u$.

COROLlary 2.1. For any measurable set $S \in U(0)$ let $S_{1}, \ldots, S_{J}$ be its preimage: $S_{j}=\left\{w \in I_{j} \mid \varphi_{j}(w) \in S\right\}$. Then

$$
\int_{S} d u=\sum_{j=1}^{J} \int_{S_{j}} p_{j}(v) d v .
$$

We prove Theorem 1 in Appendix A.

According to the terminology, introduced by Vershik, preservation of the Lebesgue measure means that $T$ is a polymorphism. Having in mind this motivation, below we study polymorphisms from dynamical point of view.

Example. Here is an example of a polymorphism associated with separatrix crossings. To have simple formulas we consider the case when the areas $A_{ \pm}(\tau)$ of the domains $D_{ \pm}(\tau)$ are piecewise linear functions of $\tau$.

In what follows $A_{*}, \hat{\tau}, \tau_{1}, d, \gamma_{0}, \gamma_{1}, \gamma_{3}$ are positive constants, and $\gamma_{2}$ is a non-negative constant, $\tau_{1}+d<\hat{\tau}, \gamma_{2}<\gamma_{1}, \gamma_{3}<\gamma_{1}$. Assume that at $\tau=0$ both areas $A_{ \pm}$have the same value $A_{*}$. Assume that for $0 \leq \tau \leq \tau_{1}$ both functions $A_{ \pm}(\tau)$ grow linearly with the same rate $\gamma_{0}: A_{ \pm}^{\prime}(\tau)=\gamma_{0}$. For $\tau_{1} \leq \tau \leq \hat{\tau}-d$ the function $A_{+}(\tau)$ decays linearly with the rate $\gamma_{1}: A_{+}^{\prime}(\tau)=-\gamma_{1}$, and for $\hat{\tau}-d \leq \tau \leq \hat{\tau}$ it decays linearly with the rate $\gamma_{2}: A_{+}^{\prime}(\tau)=-\gamma_{2}$. For $\tau_{1} \leq \tau \leq \hat{\tau}$ the function $A_{-}(\tau)$ decays linearly with the rate $\gamma_{3}: A_{-}^{\prime}(\tau)=-\gamma_{3}$. The constants are such that $A_{ \pm}(\hat{\tau})=A_{ \pm}(0)=A_{*}$, i.e.,

$$
\gamma_{0} \tau_{1}-\gamma_{1}\left(\hat{\tau}-d-\tau_{1}\right)-\gamma_{2} d=0 \quad \text { and } \quad \gamma_{0} \tau_{1}-\gamma_{3}\left(\hat{\tau}-\tau_{1}\right)=0 .
$$

Consider the motion of phase points with some initial, at $\tau=0$, action $\mathcal{I} \in\left[A_{*}, C\right]$, $C=A_{*}+2 \gamma_{0} \tau_{1}$. At $\tau=0$ these phase points are placed in the domain $D_{0}(0)$. During the 
motion they cross the separatrix at some moment of time and are captured into $D_{+}$and $D_{-}$. Then they cross the separatrix again and return into $D_{0}$ with the values of the action $\mathcal{I}_{+}$ (for phase points captured into $D_{+}$) and $\mathcal{I}_{-}$(for phase points captured into $D_{-}$). The probability of capture into each of the domains $D_{ \pm}(\tau)$ is $1 / 2$. Description of the motion in the adiabatic approximation leads to the following formulas (we omit straightforward calculations):

$$
\mathcal{I}_{+}= \begin{cases}A_{*}+\frac{\left(\mathcal{I}-A_{*}\right)\left(\gamma_{3}+\gamma_{2}\right)}{2 \gamma_{2}} & \text { if } \mathcal{I} \in\left[A_{*}, A_{*}+2 \gamma_{2} d\right], \\ A_{*}+\frac{\left(\mathcal{I}-A_{*}\right)\left(\gamma_{3}+\gamma_{1}\right)}{2 \gamma_{1}}+\frac{\gamma_{3}\left(\gamma_{1}-\gamma_{2}\right) d}{\gamma_{1}} & \text { if } \mathcal{I} \in\left[A_{*}+2 \gamma_{2} d, C\right],\end{cases}
$$

and

$$
\mathcal{I}_{-}= \begin{cases}A_{*}+\frac{\left(\mathcal{I}-A_{*}\right)\left(\gamma_{3}+\gamma_{2}\right)}{2 \gamma_{3}} & \text { if } \mathcal{I} \in\left[A_{*}, A_{*}+2 \gamma_{3} d\right], \\ A_{*}+\frac{\left(\mathcal{I}-A_{*}\right)\left(\gamma_{3}+\gamma_{1}\right)}{2 \gamma_{3}}+\left(\gamma_{2}-\gamma_{1}\right) d & \text { if } \mathcal{I} \in\left[A_{*}+2 \gamma_{3} d, C\right] .\end{cases}
$$

These formulas define a polymorphism $T$. The domain of $T$ is $\left[A_{*}, C\right] ; T$ maps $\mathcal{I}$ to $\mathcal{I}_{+}$ and $\mathcal{I}_{-}$with probabilities $1 / 2$.

Introducing the new notation

$$
x=\frac{\mathcal{I}-A_{*}}{C-A_{*}}, \quad s=\frac{d\left(\gamma_{2}+\gamma_{3}\right)}{C-A_{*}}, \quad \alpha=\frac{2 \gamma_{2}}{\gamma_{2}+\gamma_{3}}, \quad \beta=\frac{2 \gamma_{3}}{\gamma_{2}+\gamma_{3}}
$$

we arrive at the following equations, determining $T$ :

$$
\begin{gathered}
x \in[0,1] \text { is transformed to } \varphi_{1}(x) \text { or to } \varphi_{2}(x) \text { with probabilities } 1 / 2, \\
\varphi_{1}= \begin{cases}x / \beta & \text { if } 0 \leq x \leq \beta s, \\
\frac{1-s}{1-\beta s} x+\frac{s-\beta s}{1-\beta s} & \text { if } \beta s \leq x \leq 1,\end{cases} \\
\varphi_{2}= \begin{cases}x / \alpha & \text { if } 0 \leq x \leq \alpha s, \\
\frac{1-s}{1-\alpha s} x+\frac{s-\alpha s}{1-\alpha s} & \text { if } \alpha s \leq x \leq 1 .\end{cases}
\end{gathered}
$$

The coefficients $\alpha$ and $\beta$ enter these formulas symmetrically and $\alpha+\beta=2$. Hence we can assume that $0<\beta \leq 1$. We have a two-parametric family of polymorphisms

$$
T_{\beta, s}, \quad 0<\beta \leq 1,0<s<1,(2-\beta) s<1 .
$$

\section{Basic definitions}

In this section we define a specific class of polymorphisms of an interval with the Lebesgue measure. Below without loss of generality we assume that this interval is [0, 1]. According to argument from $\$ 2$ random multi-valued maps, responsible for jumps of the adiabatic invariant at the moments of the separatrix crossing are polymorphisms from this class. Here an interesting question appears. Which polymorphisms, defined in $\$ 3$, can be generated by Hamiltonian systems from $\$ 2$ ?

We plan to discuss this question elsewhere. The majority of definitions and statements from this section have natural analogs in the general situation, [7, 8].

\section{CAMBRIDGE JDURNALS}


3.1. Multivalued self-maps of an interval. Let the interval $[0,1]$ be presented as the union

$$
[0,1]=\bigcup_{j=1}^{J} I_{j}, \quad I_{j}=\left[a_{j}, b_{j}\right] .
$$

The intervals $I_{j}$ can have non-trivial pairwise intersections. For example, it can happen that all $I_{j}$ equal $[0,1]$. Below, $j$ always takes values from the set $\{1, \ldots, J\}$. For any $j$ consider the functions

$$
\varphi_{j}: I_{j} \rightarrow[0,1], \quad p_{j}: I_{j} \rightarrow[0,1] .
$$

We assume that for any $j$ the functions $\varphi_{j}$ and $p_{j}$ are smooth or piecewise smooth inside $I_{j}$.

For any $x \in[0,1]$ we put

$$
V(x)=\left\{j \mid x \in I_{j}\right\} .
$$

We assume that the following conditions hold.

$\mathbf{P}$ (Probability) $\sum_{j \in V(x)} p_{j}(x)=1$ for any $x \in[0,1]$.

M (Monotonicity) the functions $\varphi_{j}$ are strictly monotone on $I_{j}$.

According to $\mathbf{M}$ there exist the inverse functions $\psi_{j}=\varphi_{j}^{-1}$ which are smooth or piecewise smooth on $K_{j}=\varphi_{j}\left(I_{j}\right)$.

Consider the following dynamical system $T$ on $[0,1]$. Any point $x \in I_{j}$ is mapped to $\varphi_{j}(x)$ with probability $p_{j}(x)$. We write

$$
T=\left(\varphi_{1}, \ldots, \varphi_{J} ; p_{1}, \ldots, p_{J} ; I_{1}, \ldots, I_{J}\right)
$$

or (in shorter notation) $T=(\varphi ; p ; I)$.

3.2. The operator $W_{T}$. Consider the space $L_{2}=L_{2}([0,1], d x)$ of functions, square integrable on $[0,1]$ with respect to the Lebesgue measure $d x$. Below we use the notation $\langle$,$\rangle for the corresponding scalar product and \|\cdot\|$ for the corresponding norm. In a standard way we define the Perron-Frobenius operator

$$
W_{T}: L_{2} \rightarrow L_{2}, \quad f \mapsto W_{T} f .
$$

For any $y \in[0,1]$ we put

$$
V^{*}(y)=\left\{j \mid y \in \varphi_{j}\left(I_{j}\right)\right\} .
$$

Then $\left\{\psi_{j}(y) \mid j \in V^{*}(y)\right\}$ is the set of all preimages of the point $y$ with respect to $T$. By definition

$$
W_{T} f(y)=\sum_{j \in V^{*}(y)} p_{j} \circ \psi_{j}(y)\left|\psi_{j}^{\prime}(y)\right| f \circ \psi_{j}(y) .
$$

For any measurable set $\Omega \subset[0,1]$ we have

$$
\int_{\Omega} W_{T} f(y) d y=\sum_{j=1}^{J} \int_{\varphi_{j}^{-1}(\Omega)} p_{j}(x) f(x) d x .
$$

The positive cone

$$
L_{2}^{+}=\left\{\rho \in L_{2} \mid \rho \geq 0\right\}
$$

can be associated with the space of densities of finite measures on $[0,1]$. We have the obvious inclusion

$$
W_{T}\left(L_{2}^{+}\right) \subset L_{2}^{+} \text {. }
$$

\section{CAMBRIDGE JDURNALS}


3.3. Polymorphisms. Below we assume that the Lebesgue measure is invariant.

L (Lebesgue) $W_{T} 1=1$.

Equation (3.1) implies that

$$
\sum_{j \in V^{*}(y)} p_{j} \circ \psi_{j}(y)\left|\psi_{j}^{\prime}(y)\right|=1
$$

COROLlary 3.1. For any $y \in[0,1] V^{*}(y) \neq \emptyset$.

Definition 3.1. Any map $(\varphi ; p ; I)$, satisfying $\mathbf{P}, \mathbf{M}$, and $\mathbf{L}$, will be said to be a polymorphism.

A polymorphism can be regarded as a multivalued self-map of an interval preserving the Lebesgue measure.

The following construction shows a connection between Vershik's definition of a polymorphism (see the introduction) and Definition 3.1. Suppose that $(\varphi ; p ; I)$ is a polymorphism in the sense of Definition 3.1. Let $v$ be the following measure, supported on the graphs of the functions $\varphi_{j}$. For any $S \subset[0,1] \times[0,1]$ let $\chi_{S}:[0,1] \times[0,1] \rightarrow \mathbb{R}$ be its indicator:

$$
\chi_{S}(x, y)= \begin{cases}0 & \text { if }(x, y) \notin S \\ 1 & \text { if }(x, y) \in S\end{cases}
$$

Then by definition

$$
v(S)=\sum_{j=1}^{J} \int_{I_{j}} p_{j}(x) \chi_{S}\left(x, \varphi_{j}(x)\right) d x .
$$

We have an obvious proposition.

PROPOSITION 3.1. The measure v satisfies (1.2).

3.4. An adjoint polymorphism. For any polymorphism $T=(\varphi ; p ; I)$ we put

$$
K_{j}=\varphi_{j}\left(I_{j}\right), \quad q_{j}(y)=p_{j} \circ \psi_{j}(y)\left|\psi_{j}^{\prime}(y)\right| .
$$

PROPOSITION 3.2. $(\psi ; q ; K)$ is a polymorphism.

Proof. The equation $[0,1]=\bigcup_{j=1}^{J} K_{j}$ follows from Corollary 3.1. Condition M holds because $\varphi_{j}$ are smooth. We have

$$
\begin{aligned}
& \sum_{j=1}^{J} q_{j}(y)=\sum_{j=1}^{J} p_{j} \circ \psi_{j}(y)\left|\psi_{j}^{\prime}(y)\right|=1, \\
& \sum_{j=1}^{J} q_{j} \circ \varphi_{j}(x)\left|\varphi_{j}^{\prime}(x)\right|=\sum_{j=1}^{J} p_{j}(x)=1 .
\end{aligned}
$$

Hence Conditions $\mathbf{P}$ and $\mathbf{L}$ also hold.

In fact it was sufficient to note that the passage from $(\varphi ; p ; I)$ to $(\psi ; q ; K)$ corresponds to the exchange of left and right in diagram (1.1).

We say that $(\psi ; q ; K)$ is adjoint to $T:(\psi ; q ; K)=T^{*}$. Obviously $T^{* *}=T$.

\section{CAMBRIDGE}


PROPOSITION 3.3. The operator $W_{T^{*}}$ is adjoint to $W_{T}$ with respect to the $L_{2}$-scalar product: $W_{T^{*}}=W_{T}^{*}$.

Proof. Straightforward computation.

COROLlary 3.2. For any polymorphism $T$ we have $W_{T}^{*} 1=1$.

3.5. Structures. Condition $\mathbf{L}$ combined with (3.2) and Corollary 3.2 mean that $W_{T}$ is a Markov (bistochastic) operator, i.e., $W_{T}$ satisfies the following conditions.

(1) $W_{T}\left(L_{2}^{+}\right) \subset L_{2}^{+}$.

(2) $W_{T} 1=W_{T}^{*} 1=1$.

PROPOSITION 3.4. The operator $W_{T}$ is a contraction on $L_{2}$, i.e.,

$$
\left\|W_{T} f\right\| \leq\|f\| \quad \text { for any } f \in L_{2}([0,1]) .
$$

Proof. Due to (1) and the equation $W_{T} 1=1$ we can use the Jensen inequality:

$$
\left(W_{T} f(x)\right)^{2} \leq W_{T} f^{2}(x) .
$$

Therefore

$$
\begin{aligned}
\left\|W_{T} f\right\|^{2} & =\int_{0}^{1}\left(W_{T} f(x)\right)^{2} d x \leq \int_{0}^{1} W_{T} f^{2}(x) d x \\
& =\left\langle W_{T} f^{2}, 1\right\rangle=\left\langle f^{2}, W_{T}^{*} 1\right\rangle=\left\langle f^{2}, 1\right\rangle=\|f\|^{2} .
\end{aligned}
$$

The set $\mathcal{P}$ of polymorphisms obviously admits the natural structure of a semigroup with involution $*$. The set $\mathcal{P}$ is convex: for any two polymorphisms $F=(\varphi ; p ; I)$ and $G=(\psi ; q ; K)$ and $\alpha \in[0,1]$ there is a polymorphism

$$
\alpha F+(1-\alpha) G:=(\varphi, \psi ; \alpha p,(1-\alpha) q ; I, K)
$$

The boundary points of this family are naturally identified with $G$ and $F$.

3.6. Mixing and ergodicity. Let $\Lambda \subset L_{2}$ be the space of $W_{T}$-invariant functions. Then by the von Neumann ergodic theorem for contracting operators [3] for any $f \in L_{2}$ the sequence

$$
\frac{1}{N} \sum_{n=0}^{N-1} W_{T}^{n} f
$$

converges in $L_{2}$ to $\pi(f)$, where $\pi: L_{2} \rightarrow \Lambda$ is the orthogonal projection.

Definition 3.2. The polymorphism $T=(\varphi ; p ; I)$ is said to be ergodic if any fixed point of $W_{T}$ is a constant: $\Lambda=\operatorname{span}(1)$.

The polymorphism $T$ is said to be mixing if for any $f \in L_{2}$ we have

$$
W_{T}^{n} f \rightarrow \bar{f}=\langle 1, f\rangle \text { in the weak } L_{2} \text {-topology as } n \rightarrow \infty \text {. }
$$

\section{CAMBRIDGE JDURNALS}


If $T$ is ergodic, sequence (3.4) converges to $\pi(f)=\bar{f}$.

If $T$ is mixing, it is ergodic. The inverse is not true: take for example $J=1$,

$$
T=(\varphi ; 1 ;[0,1]), \quad \varphi(x)=x+\omega \bmod 1, \quad \omega=\text { const } \in \mathbb{R} \backslash \mathbb{Q} .
$$

If $T$ is mixing, $T^{*}$ is also mixing. Indeed, $\lim _{n \rightarrow \infty}\left\langle W_{T}^{n} f, g\right\rangle=\bar{f} \bar{g}$ for any $f, g \in L_{2}$ if and only if for any $f, g \in L_{2} \lim _{n \rightarrow \infty}\left\langle W_{T}^{* n} f, g\right\rangle=\bar{f} \bar{g}$.

Example. Consider the polymorphism $T=\left(\varphi_{1}, \varphi_{2} ; 1 / 2,1 / 2 ;[0,1],[0,1]\right)$,

$$
\varphi_{1}=x / 2, \quad \varphi_{2}=x / 2+1 / 2 .
$$

It is easy to check that

$$
T^{*}=(\varphi ; 1 ;[0,1]), \quad \varphi(x)=2 x \bmod 1 .
$$

Then for any $f \in L_{2}$

$$
\begin{gathered}
W_{T} f(x)= \begin{cases}f(2 x) & \text { if } x \in(0,1 / 2), \\
f(2 x-1) & \text { if } x \in(1 / 2,1),\end{cases} \\
W_{T^{*}} f(x)=\frac{1}{2}\left(f\left(\frac{x}{2}\right)+f\left(\frac{x+1}{2}\right)\right) .
\end{gathered}
$$

This implies (for example with the help of the Fourier expansions) that both $T$ and $T^{*}$ are mixing. The second statement is a standard fact from ergodic theory.

4. The case $I_{1}=\cdots=I_{J}=[0,1]$

A priori it is not obvious that conditions $\mathbf{P}, \mathbf{M}$, and $\mathbf{L}$ can be easily satisfied simultaneously. In this section we show that these conditions are quite flexible and the set of polymorphisms is sufficiently rich.

4.1. General remarks. Let $T=(\varphi ; p ; I)$ be a polymorphism. If all functions $\varphi_{j}$ are increasing, $T$ is said to be increasing.

Consider the case

$$
I_{1}=\cdots=I_{J}=\varphi_{1}\left(I_{1}\right)=\cdots=\varphi_{J}\left(I_{J}\right)=[0,1] .
$$

For such polymorphisms we use the shorter notation $T=(\varphi ; p)$. In this case $V(x)=$ $V^{*}(y)=\{1, \ldots, J\}$ for all $x, y \in[0,1]$.

If $T=(\varphi ; p)$ is an increasing polymorphism, for all $j$ we have $\varphi_{j}^{\prime} \geq 0, \varphi_{j}(0)=0$, and $\varphi_{j}(1)=1$. In this case, for any $x \in[0,1],(3.3)$ takes the form

$$
\sum_{j=1}^{J} p_{j} \circ \psi_{j}(y) \psi_{j}^{\prime}(y)=1 .
$$

Putting $P_{j}(x)=\int_{0}^{x} p_{j}(\xi) d \xi$, we obtain

$$
\sum_{j=1}^{J} P_{j} \circ \psi_{j}(y)=y .
$$

\section{CAMBRIDGE JDURNALS}




\subsection{Construction of $\varphi_{J}$.}

Proposition 4.1. Given $C^{1}$-smooth $\varphi_{1}, \ldots, \varphi_{J-1}:[0,1] \rightarrow[0,1], \varphi_{j}^{\prime}>0, \varphi_{j}(0)=0$, $\varphi_{j}(1)=1$ and continuous $p_{1}, \ldots, p_{J}:[0,1] \rightarrow[0,1]$, such that for some $c_{1}, c_{2}, c_{3} \in$ $[0,1]$

$$
\sum_{j=1}^{J} p_{j}=1, \quad \sum_{j=1}^{J-1} p_{j} \circ \psi_{j} \cdot \psi_{j}^{\prime}<1-c_{1}, c_{2}<p_{J}<c_{3},
$$

then there exists a unique $\varphi_{J}:[0,1] \rightarrow[0,1]$ such that $(\varphi ; p)$ is a polymorphism, $\varphi_{J}$ is $C^{1}$-smooth, $c_{2}<\varphi_{J}^{\prime}<c_{3} / c_{2}, \varphi_{J}(0)=0$, and $\varphi_{J}(1)=1$.

Proof. By (4.3) the function $P_{J}^{-1}$ is $C^{1}$-smooth and $1 / c_{3}<\left(P_{J}^{-1}\right)^{\prime}<1 / c_{2}$. By using (4.2) we obtain

$$
\psi_{J}(y)=P_{J}^{-1}\left(y-\sum_{j=1}^{J-1} P_{j} \circ \psi_{j}(y)\right) .
$$

According to (4.3),

$$
c_{1} / c_{3}<\psi_{J}^{\prime}<1 / c_{2}
$$

We also have

$$
\psi_{J}(0)=P_{J}^{-1}(0)=0, \quad \psi_{J}(1)=P_{J}^{-1}\left(1-\sum_{j=1}^{J-1} P_{j}(1)\right)=P_{J}^{-1}\left(P_{J}(1)\right)=1 .
$$

Proposition 4.2. Let $(\varphi ; p)$ be an increasing polymorphism. Suppose that $x \in[0,1]$ is a point such that $\varphi_{1}(x)=\cdots=\varphi_{J-1}(x)=x$. Then $\varphi_{J}(x)=x$.

Indeed, by using (4.4) and (4.2), we obtain that $\psi_{1}(x)=\cdots=\psi_{J-1}(x)=x$ implies that $\varphi_{J}(x)=x$.

\subsection{Construction of $p$.}

Proposition 4.3. Given smooth increasing functions $f, g:[0,1] \rightarrow[0,1]$ such that $f(0)=g(0)=0, \quad f(1)=g(1)=1, \quad f(x)<x<g(x)$ for $x \in(0,1) . \quad$ Consider the function $\dagger$

$$
\begin{aligned}
\vartheta(x) & =f(x)-g^{-1} \circ f(x)+f \circ g^{-1} \circ f(x)-g^{-1} \circ f \circ g^{-1} \circ f(x)+\cdots \\
& =\sum_{n=1}^{+\infty}\left(g \circ\left(g^{-1} \circ f\right)^{n}(x)-\left(g^{-1} \circ f\right)^{n}(x)\right) .
\end{aligned}
$$

Suppose that $\vartheta^{\prime} \in(0,1)$.

Then $(f, g ; p, 1-p)$ is an increasing polymorphism, where $p=\vartheta^{\prime}$.

Proof. We put in (4.2) $J=2, P_{1}(x)=P(x), P_{2}(x)=x-P(x), \psi_{1}=f^{-1}, \psi_{2}=g^{-1}$, and $y=f(x)$. Then we have

$$
P(x)-P \circ g^{-1} \circ f(x)=f(x)-g^{-1} \circ f(x) .
$$

$\dagger$ Series (4.5) is alternating and absolute values of its terms monotonically tend to zero. Therefore it converges.

\section{CAMBRIDGE}


Plugging in this equation $g^{-1} \circ f(x), g^{-1} \circ f \circ g^{-1} \circ f(x), \ldots$ instead of $x$ and performing summation, we get

$$
P(x)-P(0)=\vartheta(x) .
$$

Here we have used the fact that the sequence

$$
f(x), g^{-1} \circ f(x), f \circ g^{-1} \circ f(x), g^{-1} \circ f \circ g^{-1} \circ f(x), \ldots
$$

tends to zero for any $x \in[0,1]$. Hence $p(x)=P^{\prime}(x)=\vartheta^{\prime}(x)$.

Now we present a condition necessary for the inclusion $\vartheta^{\prime} \in[0,1]$.

Proposition 4.4. Suppose that $\vartheta^{\prime}(x)$ is bounded and

$$
\lim _{n \rightarrow+\infty} \frac{d}{d x}\left(g^{-1} \circ f\right)^{-n}(x)=0 \quad \text { for any } x \in(0,1) .
$$

Then the function

$$
\Theta(x)=\sum_{n=-\infty}^{+\infty}\left(g \circ\left(g^{-1} \circ f\right)^{n}(x)-\left(g^{-1} \circ f\right)^{n}(x)\right)
$$

does not depend on $x$.

Proof. For any $x \in(0,1)$ we have

$$
\lim _{n \rightarrow+\infty} \vartheta \circ\left(g^{-1} \circ f\right)^{-n}(x)=\Theta(x) .
$$

Moreover, by (4.6), we have the same equation for the derivatives:

$$
\lim _{n \rightarrow+\infty} \frac{d}{d x} \vartheta \circ\left(g^{-1} \circ f\right)^{-n}(x)=\frac{d}{d x} \Theta(x) .
$$

Given any point $x_{0} \in(0,1)$ we put $x_{n}=\left(g^{-1} \circ f\right)^{-n}\left(x_{0}\right)$. Then

$$
\left.\frac{d}{d x}\right|_{x=x_{0}} \vartheta \circ\left(g^{-1} \circ f\right)^{-n}(x)=\vartheta^{\prime}\left(x_{k}\right) \cdot \frac{d}{d x}\left(g^{-1} \circ f\right)^{-n}\left(x_{0}\right) \text {. }
$$

In the limit $n \rightarrow+\infty$ this quantity equals $\Theta^{\prime}\left(x_{0}\right)$. By using (4.6) and boundedness of $\vartheta^{\prime}$ we obtain $\Theta^{\prime}=0$.

\section{Some non-ergodic polymorphisms}

A generic polymorphism should be expected to be ergodic and even mixing. However there are some exceptions. In this section we present two mechanisms which prevent ergodicity.

Let $T=(\varphi ; p)$ be an increasing polymorphism such that for some point $x_{0} \in$ $(0,1) \varphi_{1}\left(x_{0}\right)=\cdots=\varphi_{J-1}\left(x_{0}\right)=x_{0}$. Then, by Proposition 4.2, $\varphi_{J}\left(x_{0}\right)=x_{0}$ and the intervals $\left[0, x_{0}\right]$ and $\left[x_{0}, 1\right]$ are invariant. Such a polymorphism is not ergodic because $W_{T} f=f$ for any $f$ equal to constants (may be distinct) on $\left(0, x_{0}\right)$ and $\left(x_{0}, 1\right)$.

This example is in a sense trivial because the dynamics, restricted to any of the intervals $\left(0, x_{0}\right)$ and $\left(x_{0}, 1\right)$ can be ergodic. Now we present a less obvious example of a 'nonergodic', in fact, 'non-chaotic' polymorphism.

\section{CAMBRIDGE}


Proposition 5.1. There exist $f, p:[0,1] \rightarrow[0,1]$ such that the following hold.

(a) $f \in C^{\infty}([0,1])$.

(b) $f(x)<x$ for any $x \in(0,1)$.

(c) $f(0)=0, f(1)=1$.

(d) $\left.f^{\prime}\right|_{[0,1]}>0$.

(e) $0<p(x)<1$ for any $x \in(0,1)$.

(f) $\left(f, f^{-1} ; p, 1-p\right)$ is a polymorphism.

Any increasing polymorphism $\left(f, f^{-1} ; p, 1-p\right)$ from Proposition 5.1 presents another mechanism of the absence of ergodicity. Indeed, let $[\alpha, \beta)$ be a 'fundamental' semi-interval, i.e.,

$$
f^{n}([\alpha, \beta)) \cap f^{m}([\alpha, \beta))=\emptyset \quad \text { for all integer } m \neq n \text {, and } \bigcup_{n \in \mathbb{Z}} f^{n}([\alpha, \beta))=(0,1) .
$$

For any $\rho_{0} \in L_{2}$, supp $\rho_{0} \subset[\alpha, \beta]$, obviously

$$
\rho:=\sum_{n=-\infty}^{\infty} \rho_{0} \circ f^{n} \in L_{2} \quad \text { and } \quad W_{T} \rho=\rho .
$$

\section{Proof of Proposition 5.1.}

LEMMA 5.1. Given $\lambda>0$ there exists a function $h: \mathbb{R} \rightarrow(0,1)$ such that the following hold.

(1) $h \in C^{\infty}(\mathbb{R})$.

(2) $h^{\prime}>0, h(-\infty)=0, h(+\infty)=1$.

(3) The function $h-1 / 2$ is odd.

(4) $h^{\prime}(y) \geq h^{\prime}(y-1)$ for $y \leq 0$.

(5) $\sum_{n=-\infty}^{+\infty}(h(2 n+y)-h(2 n-1+y))$ does not depend on $y$.

(6) $h^{\prime}(y-1) / h^{\prime}(y)=e^{\lambda}(1+O(1 / y))$ for $y \rightarrow+\infty$.

Proposition 5.1 follows from Lemma 5.1. Indeed, for any $x \in(0,1)$ we put $f(x)=$ $h\left(-1+h^{-1}(x)\right)$. For any natural $n$ we obtain

$$
f^{n}(x)=h\left(-n+h^{-1}(x)\right) .
$$

Then assertions (a), (b), and (c) obviously hold. We have to check (d) only for $x=0$ and $x=1$. For $x$ close to 1 ,

$$
f^{\prime}(x)=\frac{h^{\prime}(-1+y)}{h^{\prime}(y)}=e^{\lambda}(1+O(1 / y)), \quad y=h^{-1}(x) .
$$

Hence $\lim _{x \rightarrow 1} f^{\prime}(x)=e^{\lambda}$. We have $\lim _{x \rightarrow 0} f^{\prime}(x)=e^{-\lambda}$ because $h^{\prime}$ is odd.

To check (e), we note that, by condition (5),

$$
\sum_{n=-\infty}^{+\infty}\left(h^{\prime}(2 n+y)-h^{\prime}(2 n-1+y)\right)=0 .
$$

(The series converges according to condition (6).) As in Proposition 4.3 we define $p=\vartheta^{\prime}$ (see (4.5)). The function $\vartheta$ in the case $g=f^{-1}$ has the form

$$
\vartheta(x)=\sum_{n=1}^{+\infty}\left(f^{2 n-1}(x)-f^{2 n}(x)\right)=\sum_{n=1}^{+\infty}\left(h\left(1-2 n+h^{-1}(x)\right)-h\left(-2 n+h^{-1}(x)\right)\right) .
$$

\section{CAMBRIDGE}


Therefore

$$
\vartheta^{\prime}(x)=\sum_{n=1}^{+\infty} \frac{h^{\prime}(1-2 n+y)-h^{\prime}(-2 n+y)}{h^{\prime}(y)}, \quad y=h^{-1}(x) .
$$

Now we check (e). By Proposition 4.3, $p=\vartheta^{\prime}$. Hence we have to prove the inequality

$$
0<\vartheta^{\prime}(x)<1 \text { for } x \in(0,1) .
$$

We put $y=h^{-1}(x)$. Then

$$
\vartheta^{\prime}(x)=\frac{h^{\prime}(-1+y)-h^{\prime}(-2+y)+h^{\prime}(-3+y)-h^{\prime}(-4+y)+\cdots}{h^{\prime}(y)} .
$$

First, suppose that $y \leq 0$. The series in the numerator is alternating and by condition (4) absolute values of its terms monotonically decrease. Hence its sum $S$ satisfies $0<S<$ $h^{\prime}(-1+y)$. Since $h^{\prime}(y)>h^{\prime}(-1+y)$, we obtain (5.2).

Now suppose that $y=h^{-1}(x)>0$. Then by using the fact that $h^{\prime}$ is odd, we have

$$
\vartheta^{\prime}(x)=\frac{h^{\prime}(1-y)-h^{\prime}(2-y)+h^{\prime}(3-y)-h^{\prime}(4-y)+\cdots}{h^{\prime}(-y)} .
$$

By (5.1), we get

$$
\vartheta^{\prime}(x)=\frac{h^{\prime}(-y)-h^{\prime}(-1-y)+h^{\prime}(-2-y)-h^{\prime}(-3-y)+\cdots}{h^{\prime}(-y)} .
$$

The series in the numerator is alternating and absolute values of its terms monotonically decrease. Hence its sum $S$ satisfies $0<S<h^{\prime}(-y)$. This implies (5.2).

We prove Lemma 5.1 in Appendix C.

\section{6. $L_{2}$-norm and $W_{T}$}

The main result of this section is Theorem 3 which shows that in some cases mixing property follows from ergodicity.

6.1. On the difference $\|f\|^{2}-\left\|W_{T} f\right\|^{2}$. Let $T=(\varphi ; p)$ be a polymorphism. Then by (3.1)

$$
W_{T} f(x)=\sum_{j=1}^{J} q_{j}(x) f \circ \psi_{j}(x), \quad q_{j}(x)=p_{j} \circ \psi_{j}(x)\left|\psi_{j}^{\prime}(x)\right| .
$$

Proposition 6.1. For any $f \in L_{2}$,

$$
\begin{gathered}
\|f\|^{2}-\left\|W_{T} f\right\|^{2}=\frac{1}{2} \sum_{j, k} B_{j k}(f), \\
B_{j k}(f)=\int_{0}^{1} q_{j}(y) q_{k}(y)\left(f \circ \psi_{j}(y)-f \circ \psi_{k}(y)\right)^{2} d y .
\end{gathered}
$$

COROllary 6.1. Suppose that $q_{j}, j=1, \ldots, J$, do not vanish almost everywhere. Then we have the following.

(a) The equation $\left\|W_{T} f\right\|=\|f\|$ implies that $f \circ \psi_{j}$ does not depend on $j$.

(b) The equation $W_{T} f=f$ implies that $f \circ \psi_{j}=f$ for all $j$.

\section{CAMBRIDGE}


COROLlaRy 6.2. Suppose that $q_{j}, j=1, \ldots, J$, do not vanish almost everywhere. Then $T=(\varphi ; p)$ is ergodic if and only if $T^{*}$ is ergodic.

Indeed, the equations $f \circ \psi_{j}=f$ and $f \circ \varphi_{j}=f$ are equivalent.

Let $H_{j}, H_{j k}(j \neq k)$ be the spaces defined as follows:

$$
\begin{gathered}
H_{j}=\left\{f \in L_{2} \mid f=f \circ \psi_{j}\right\}, \\
H_{j k}=\left\{f \in L_{2} \mid f \circ \psi_{j}=f \circ \psi_{k}\right\} .
\end{gathered}
$$

COROllary 6.3. Suppose that $q_{j}, j=1, \ldots, J$ do not vanish almost everywhere and $\bigcap_{j=1}^{J} H_{j}=\operatorname{span}(1)$. Then $T$ is ergodic.

Proof of Proposition 6.1 is based on a direct calculation. By using the equations $\sum_{j} p_{j}=\sum_{j} q_{j}=1$, we get

$$
\begin{aligned}
&\|f\|^{2}=\int \sum_{j} p_{j}(x) f^{2}(x) d x=\int \sum_{j} q_{j}(y) f^{2} \circ \psi_{j}(y) d y \\
&=\int \sum_{j, k} q_{k}(y) q_{j}(y) f^{2} \circ \psi_{j}(y) d y \\
&\left\|W_{T} f\right\|^{2}=\int \sum_{j, k} q_{j}(y) q_{k}(y) f \circ \psi_{j}(y) f \circ \psi_{k}(y) d y .
\end{aligned}
$$

Equation (6.1) now follows.

6.2. A lower estimate for integral (6.2). In this section we estimate integrals (6.2) in terms of $\|f\|$ provided $f$ lies in $H_{j k}^{\perp}$. The spaces $H_{j k}$, defined in (6.3), can be presented in the form

$$
H=\left\{f \in L_{2} \mid f=f \circ h_{j k}\right\}, \quad h_{j k}=\psi_{j} \circ \varphi_{k} .
$$

Below we fix a pair of indices $j \neq k$ and skip them for brevity: $H=H_{j k}$ and $h=h_{j k}$.

Consider a smooth or piecewise-smooth increasing function $h:[0,1] \rightarrow[0,1]$ such that the following hold.

(i) $\quad h(0)=0, h(1)=1$, and $h^{\prime}(x)>0$ for any $x \in(0,1)$ (if $h$ is not differentiable at $x$, $h^{\prime}(x)$ denotes right and left derivatives).

(ii) The function $h(x)-x$ does not have zeros on $(0,1)$.

(iii) $\lim _{x \rightarrow 0} h^{\prime}(x)=\lambda_{0}, \lim _{x \rightarrow 1} h^{\prime}(x)=\lambda_{1}$, where $0<\lambda_{0}<1<\lambda_{1}$ or $0<\lambda_{1}<1<\lambda_{0}$.

We define the space

$$
H=\left\{f \in L_{2} \mid f=f \circ h\right\}
$$

and its orthogonal complement $H^{\perp}$. For a positive function $Q:[0,1] \rightarrow \mathbb{R}, Q>\hat{c}>0$, consider the quadratic functional

$$
B(f)=\int_{0}^{1} Q(x)(f \circ h(x)-f(x))^{2} d x, \quad f \in L_{2} .
$$

We put

$$
c=\inf _{0 \neq f \in H^{\perp}} B(f)\|f\|^{-2}=\inf _{f \in H^{\perp},\|f\|=1} B(f) .
$$

\section{CAMBRIDGE JDURNALS}


LEMMA 6.1. The constant $c$ is positive.

Proof of Lemma 6.1 is contained in Appendix B.

COROLlary 6.4. Suppose that $q_{j}, q_{k}>c_{0}>0$ and $\psi_{j} \circ \varphi_{k}$ satisfies conditions (i)-(iii). Then there exists a constant $c>0$ such that for any $f \in L_{2}$

$$
\|f\|-\left\|W_{T} f\right\| \geq c\left\|\operatorname{pr}_{H_{j k}^{\perp}}(f)\right\|,
$$

where $\operatorname{pr}_{H_{j k}^{\perp}}: L_{2} \rightarrow H_{j k}^{\perp}$ is the orthogonal projection to $H_{j k}^{\perp}$.

\subsection{Mixing condition for $(\varphi ; p)$.}

THEOREM 2. Let $T=\left(\varphi_{1}, \ldots, \varphi_{J} ; p_{1}, \ldots, p_{J}\right), J \geq 3$ be a polymorphism such that $q_{1}, q_{2}, q_{3}>c_{0}>0$ and the functions $\psi_{1} \circ \varphi_{2}, \psi_{1} \circ \varphi_{3}$ satisfy conditions (i)-(iii). Suppose that $H_{12} \cap H_{13}=\operatorname{span}(1)$. Then $T$ is mixing.

Proof. For any $f \in L_{2}$ we will prove that $W_{T}^{n} f$ weakly tends to $\langle 1, f\rangle$ as $n \rightarrow \infty$. It is sufficient to consider the case $\langle 1, f\rangle=0$ because

$$
W_{T}(1)=1 \quad \text { and } \quad W_{T}\left(\operatorname{span}(1)^{\perp}\right) \subset \operatorname{span}(1)^{\perp} .
$$

We write $\mathcal{H}_{12}=H_{12} \cap \operatorname{span}(1)^{\perp}, \mathcal{H}_{13}=H_{13} \cap \operatorname{span}(1)^{\perp}$. Then $\mathcal{H}_{12} \cap \mathcal{H}_{13}=0$.

Let $H$ be a Hilbert space and $\Pi$ its closed subspace. For any non-zero $v \in H$ we define the angle between $v$ and $\Pi$ :

$$
\widehat{(v, \Pi)}=\arccos \left(\sup _{0 \neq w \in \Pi} \frac{\langle v, w\rangle}{\|v\|\|w\|}\right)=\arcsin \left(\inf _{0 \neq w \in \Pi^{\perp}} \frac{\langle v, w\rangle}{\|v\|\|w\|}\right) .
$$

Consider the sequence

$$
f_{0}, f_{1}, \ldots \in \operatorname{span}(1)^{\perp}, \quad f_{k}=W_{T}^{k} f .
$$

If at least one of the sequences $\left(\widehat{f_{k}, \mathcal{H}_{12}}\right)$ or $\left(\widehat{f_{k}, \mathcal{H}_{13}}\right)$ does not tend to zero, by Corollary 6.4 we have $\left\|f_{k}\right\| \rightarrow 0$. Hence it remains to consider the case when $\lim _{k \rightarrow \infty}\left(\widehat{f_{k}, \mathcal{H}_{12}}\right)=\lim _{k \rightarrow \infty}\left(\widehat{f_{k}, \mathcal{H}_{12}}\right)=0$. In this case the proof directly follows from the lemma.

Lemma 6.2. Let $\Pi_{1}, \Pi_{2} \subset H, \Pi_{1} \cap \Pi_{2}=0$ be two closed subspaces in the Hilbert space $H$. Suppose that the sequence $v_{1}, v_{2}, \ldots \in B_{c}$ is such that $\left\|v_{k}\right\| \leq c, c>0$, and

$$
\lim _{k \rightarrow \infty}\left(\widehat{v_{k}, \Pi_{1}}\right)=\lim _{k \rightarrow \infty}\left(\widehat{v_{k}, \Pi_{2}}\right)=0 .
$$

Then $v_{k} \rightarrow 0$ weakly as $k \rightarrow \infty$.

Proof of Lemma 6.2. For any $w \in \Pi_{1}^{\perp}$ we have

$$
\lim _{j \rightarrow \infty} \frac{\left\langle v_{j}, w\right\rangle}{\left\|v_{j}\right\|}=0 .
$$

Since $\left\|v_{j}\right\| \leq c$, this implies that

$$
\lim _{j \rightarrow \infty}\left\langle v_{j}, w\right\rangle=0 .
$$

Analogously, equation (6.6) holds for any $w \in \Pi_{2}^{\perp}$.

\section{CAMBRIDGE JDURNALS}


Since $\Pi_{1} \cap \Pi_{2}=0$ and $\Pi_{1}, \Pi_{2}$ are closed, the space $\Pi_{1}^{\perp} \oplus \Pi_{2}^{\perp}$ is dense in $H$. (Indeed, if $\overline{\Pi_{1}^{\perp} \oplus \Pi_{2}^{\perp}} \neq H$, we have $\emptyset \neq{\overline{\Pi_{1}^{\perp} \oplus \Pi_{2}^{\perp}}}^{\perp} \subset \Pi_{1} \cap \Pi_{2}$.) Hence (6.6) holds for any $w \in H$.

THEOREM 3. Let $T=(\varphi ; p)$ be an ergodic polymorphism, $q_{1}, \ldots, q_{J}>c_{0}>0$ and the functions $\psi_{j} \circ \varphi_{k}$ satisfy conditions $(i)$-(iii). Then $T$ is mixing.

Proof. For any $f \in L_{2}$ we will show that the sequence $f_{k}=W_{T}^{k} f$ weakly tends to $\langle 1, f\rangle$. It is sufficient to consider the case $\langle 1, f\rangle=0$.

Consider an arbitrary weakly converging subsequence $f_{k_{l}} \rightarrow \hat{f}$. Then for any $j, s \in$ $\{1, \ldots, J\}$ and $w \in H_{j s}^{\perp}$. we have $\left\langle f_{k_{l}}, w\right\rangle \rightarrow\langle\hat{f}, w\rangle$. The last quantity is always zero (otherwise by Corollary 6.4 we have $\left\|f_{k_{l}}\right\| \rightarrow 0$ ). Hence, $\hat{f} \in H_{j s}$, i.e., $\hat{f} \circ \psi_{1}=\cdots=$ $\hat{f} \circ \psi_{J}$.

Since $\hat{f}=W_{T} \hat{f}$ we obtain

$$
\hat{f}=\sum q_{j} \hat{f} \circ \psi_{j}=\hat{f} \circ \psi_{1} \sum q_{j}=\hat{f} \circ \psi_{1} .
$$

Analogously $\hat{f}=\hat{f} \circ \psi_{j}, j=2, \ldots, J$. By using ergodicity of $T$ and the equation $\langle 1, \hat{f}\rangle=0$, we get: $\hat{f}=0$.

Thus the limit of any weakly converging subsequence of $f_{k}$ is zero. This implies that $f_{k}$ weakly converges to zero. Indeed, suppose that there exists $w \in L_{2}$ and a subsequence $f_{k_{s}}$ such that

$$
\left|\left\langle f_{k_{s}}, w\right\rangle\right|>\hat{c}>0 \text {. }
$$

Since the norms $\left\|f_{k}\right\|$ are bounded, we can choose in $f_{k_{s}}$ a weakly converging subsequence. We obtain a contradiction with (6.7) because its weak limit should be zero.

\section{Polymorphisms $T_{\beta, s}$}

In this section we study one two-parametric family of polymorphisms. The main result is that for an open set in the parameter plane these polymorphisms are mixing.

Consider the polymorphism $T_{\beta, s}=\left(\varphi_{1}, \varphi_{2} ; 1 / 2,1 / 2\right)$, where the functions $\varphi_{1,2}$ : $[0,1] \rightarrow[0,1]$ are defined in $(2.5)-(2.6)$,

$$
s, \beta \in(0,1), \quad \alpha+\beta=2, \alpha s<1 .
$$

Graphs of the functions $\varphi_{1}$ and $\varphi_{2}$ are presented in Figure 2. Note that the case $\beta=0$, $s=1 / 2$ was considered in $\$ 3.6$.

Below we assume that $\alpha, \beta$, and $s$ satisfy the following condition.

C For some $l, m \in \mathbb{N}$,

$$
\begin{gathered}
\frac{\alpha-s}{1-\beta s}<\beta^{l} \alpha^{m}<\frac{1}{\beta}, \\
\frac{\alpha}{1+s(1-\beta)}<\beta^{l} \alpha^{m}<\frac{1}{\beta} \frac{\alpha}{1+s(1-\beta)}, \\
\beta^{l} \alpha^{m}<\frac{1-s}{1-\beta} \frac{\alpha}{1+s(1-\beta)} .
\end{gathered}
$$

\section{CAMBRIDGE JDURNALS}




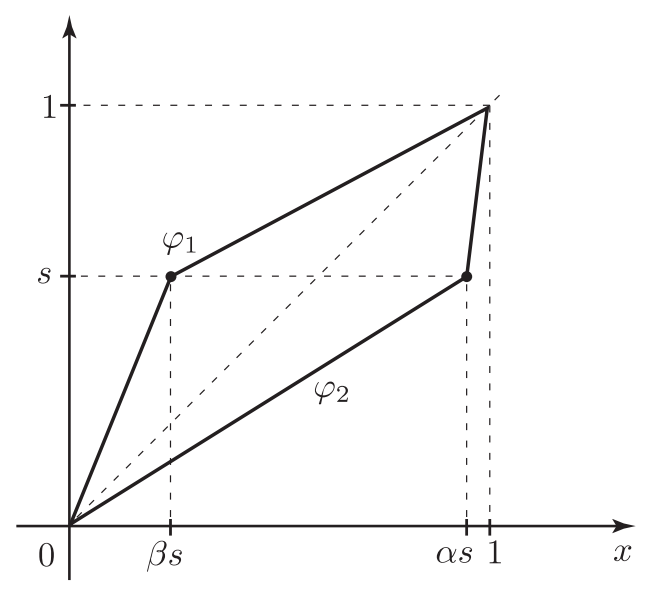

FIGURE 2. Polymorphism $T_{\beta, s}$.

If $\beta \leq s$, the inequalities are incompatible because the right-hand side of (7.4) is not greater than the left-hand side of (7.3). If $\beta>s$, inequalities (7.2)-(7.4) hold for $l=0$, $m=1$; however, $\mathbf{C}$ requires $l, m>0$.

THEOREM 4. Suppose that condition $\mathbf{C}$ holds. Then the polymorphism $T_{\beta, s}$ is mixing.

Remarks. (1) The change of variable $x \mapsto 1-x$ transforms $T_{\beta, s}$ to $T_{\beta^{\prime}, s^{\prime}}$, where

$$
\beta^{\prime}=\frac{1-\alpha s}{1-s}, \quad s^{\prime}=1-s .
$$

Hence Theorem 4 remains true if in condition $\mathbf{C}$ we replace $\alpha, \beta, s$ by $\alpha^{\prime}, \beta^{\prime}, s^{\prime}$. Note that the inequalities $\beta>s$ and $\beta^{\prime}>s^{\prime}$ are equivalent.

(2) Condition $\mathbf{C}$ obviously holds if $\beta>s$ and $\log \alpha / \log \beta$ is irrational, because in this case the numbers $\beta^{l} \alpha^{m}, l, m \in \mathbb{N}$, form a dense set on $[0, \infty)$. Hence the set of $\beta, s$, for which condition $\mathbf{C}$ holds, is open and dense in the two-dimensional domain

$$
\{(\beta, s): \beta>s \text { and conditions (7.1) hold }\} .
$$

(3) We expect that condition $\mathbf{C}$ is technical and Theorem 4 holds for all $\beta, s$, satisfying (7.1).

Proof of Theorem 4. We will prove that $T_{\beta, s}$ is ergodic. Then Theorem 3 implies that it is mixing.

Let $f \in L_{2}$ satisfy the equation $W_{T_{\beta, s}} f=f$. Then by Corollary 6.1(b) we have

$$
f=f \circ \varphi_{1}=f \circ \varphi_{2} .
$$

We will prove that (7.5) implies $f=$ constant.

The further argument is based on the following lemma. Consider the interval $(a, b) \in \mathbb{R}$ and two linear contractions $h_{a}, h_{b}:(a, b) \rightarrow(a, b)$,

$$
h_{a}(x)=\lambda_{a}(x-a)+a, \quad h_{b}(x)=\lambda_{b}(x-b)+b, 0<\lambda_{a}, \lambda_{b}<1 .
$$

\section{CAMBRIDGE}


LeMma 7.1. Suppose that $\lambda_{a}+\lambda_{b}>1$. Then any measurable function $g:(a, b) \rightarrow \mathbb{R}$, satisfying the equations $g=g \circ h_{a}=g \circ h_{b}$ equals a constant almost everywhere.

We prove the lemma in Appendix D and now return to the proof of Theorem 4. To apply Lemma 7.1 we construct two maps $\Phi_{a}, \Phi_{b}$ such that the following hold.

(a) $\Phi_{a}$ and $\Phi_{b}$ are finite compositions of $\varphi_{1}$ and $\varphi_{2}$.

(b) For some intervals $I_{a}, I_{b} \subset(0,1)$ the restrictions $\hat{\Phi}_{a}=\left.\Phi_{a}\right|_{I_{a}}$ and $\hat{\Phi}_{b}=\left.\Phi_{b}\right|_{I_{b}}$ are linear contractions.

(c) $\left.\Phi_{a}\right|_{(a, b)}=h_{a}$ and $\left.\Phi_{b}\right|_{(a, b)}=h_{b}$ for some points $a, b \in I_{a} \cap I_{b}$, where $0<\lambda_{a}$, $\lambda_{b}<1$ and $\lambda_{a}+\lambda_{b}>1$.

(d) $\bigcup_{k=-\infty}^{\infty} \varphi_{1}^{k}\left(I_{b}\right)=(0,1)$.

Conditions (a)-(c) imply that the maps $h_{a}=\left.\Phi_{a}\right|_{(a, b)}$ and $h_{b}=\left.\Phi_{b}\right|_{(a, b)}$ satisfy Lemma 7.1. Therefore any $f$, satisfying (7.5), is a constant almost everywhere on $(a, b)$ and, moreover, on $I_{a} \cup I_{b}$. By (d), the condition $\left.f\right|_{I_{b}}=$ constant implies that $f=$ constant almost everywhere on $[0,1]$.

Below it can happen that $b<a$. However we still denote the corresponding interval $(a, b)$. We put

$$
\Phi_{b}=\varphi_{2} \circ \varphi_{1}=\frac{1}{\alpha}\left(\frac{1-s}{1-\beta s} x+\frac{s-\beta s}{1-\beta s}\right), \quad I_{b}=\left(\beta s, \frac{1-\alpha \beta s}{1-s} s\right) .
$$

Since $s=\varphi_{1}(\beta s)$ and $\alpha \beta<1$, we have $(\beta s, s) \subset I_{b}$. Therefore condition (d) is satisfied.

The map $\hat{\Phi}_{b}$ is a linear contraction with

$$
\lambda_{b}=\frac{1-s}{\alpha(1-\beta s)}, \quad b=\frac{s}{1+s(1-\beta)} \in I_{b} .
$$

Proof of the inequality $\beta s<b<(1-\alpha \beta s) /(1-s) s$ is straightforward.

For $l, m \in \mathbb{N}$ satisfying (7.2)-(7.4) we put

$$
\Phi_{a}=\varphi_{1}^{l} \circ \varphi_{2}^{m} \circ \varphi_{1}
$$

Below we show that

$$
I_{a} \subset I_{b} \quad \text { and } \quad \Phi_{a}\left(I_{a}\right) \subset(0, s)
$$

Inclusions (7.6) imply

$$
\Phi_{a}=\frac{\alpha}{\omega} \Phi_{b}=\frac{1}{\omega}\left(\frac{1-s}{1-\beta s} x+\frac{s-\beta s}{1-\beta s}\right), \quad \omega=\beta^{l} \alpha^{m} .
$$

We put

$$
a=\frac{(1-\beta) s}{\omega(1-\beta s)-1+s} .
$$

Then $a \in(\beta s, s)$ because this inclusion is equivalent to (7.2). By (7.7) we obtain $\Phi_{a}(a)=a$.

The inclusion $\Phi_{a}(b) \in(\beta s, s)$ is equivalent to (7.3). We take $I_{a}$ equal to a small neighborhood of $(a, b)$. Then $I_{a} \subset I_{b}$ and $\Phi_{a}\left(I_{a}\right) \subset(\beta s, s)$. Therefore conditions (7.6) are satisfied.

\section{CAMBRIDGE}


Now conditions (a) and (b) also hold. The map $\hat{\Phi}_{b}$ is a linear contraction with

$$
\lambda_{a}=\frac{1-s}{\omega(1-\beta s)} .
$$

Finally let us turn to condition (c). Proof of the inequality $0<\lambda_{b}<1$ is straightforward. The condition $0<\lambda_{a}<1$ is equivalent to the inequality $\omega>(1-s) /(1-\beta s)$ which follows from (7.2). The condition $\lambda_{a}+\lambda_{b}>1$ is equivalent to (7.4).

Acknowledgements. The authors thank G. Piftankin and O. Zubelevich for useful discussions. The work is partially supported by RFBR grants (projects 08-01-00681-a, 09-01-00333-a) and the Program for Supporting Leading Scientific Schools (Grant NSh1312.2006.1).

\section{A. Appendix. Preservation of the measure}

In this section we prove Theorem 1. It is sufficient to prove that any map $T_{\tau_{s}}^{\tau_{s+1}}$ pulls back the measure $d u$ on $U\left(\tau_{s+1}\right)$ to the measure $d u$ on $U\left(\tau_{s}\right)$. We can assume that $\left(\tau_{s}, \tau_{s+1}\right)$ does not contain critical points of $A_{0}, A_{+}$, and $A_{-}$.

Now we will have to consider several cases, depending on signs of $A_{0}^{\prime}, A_{+}^{\prime}$, and $A_{-}^{\prime}$ on $\left(\tau_{s}, \tau_{s+1}\right)$ :

$$
\begin{array}{clll}
(+++) & A_{0}^{\prime}>0 & \text { and } & A_{ \pm}^{\prime}>0 \\
(+ \pm) & A_{0}^{\prime}>0 & \text { and } & A_{+}^{\prime} A_{-}^{\prime}<0 \\
(---) & A_{0}^{\prime}<0 & \text { and } & A_{ \pm}^{\prime}<0 \\
(- \pm) & A_{0}^{\prime}<0 & \text { and } & A_{+}^{\prime} A_{-}^{\prime}<0
\end{array}
$$

We will discuss in details the cases $(+++)$ and $(+ \pm), A_{+}^{\prime}<0$. The cases $(---)$ and $(+ \pm), A_{+}^{\prime}>0$ are completely similar to the case $(+ \pm), A_{+}^{\prime}<0$. The case $(- \pm)$ is completely similar to the case $(+++)$.

Case $(+++)$. In this case $T_{\tau_{s}}^{\tau_{s+1}}$ equals the identity on

$$
\left(0, A_{+}\left(\tau_{s}\right)\right) \sqcup\left(0, A_{-}\left(\tau_{s}\right)\right) \sqcup\left(A_{0}\left(\tau_{s+1}\right), C\right) .
$$

Hence we just need to consider the restriction $\mathcal{T}_{s}=\left.T_{\tau_{s}}^{\tau_{s+1}}\right|_{\left(A_{0}\left(\tau_{s}\right), A_{0}\left(\tau_{s+1}\right)\right)}$

$$
\mathcal{T}_{s}:\left(A_{0}\left(\tau_{s}\right), A_{0}\left(\tau_{s+1}\right)\right) \rightarrow\left(A_{+}\left(\tau_{s}\right), A_{+}\left(\tau_{s+1}\right)\right) \sqcup\left(A_{-}\left(\tau_{s}\right), A_{-}\left(\tau_{s+1}\right)\right) .
$$

Let $u_{0}$ be an arbitrary point in $\left(A_{0}\left(\tau_{s}\right), A_{0}\left(\tau_{s+1}\right)\right)$. It has then two images: $u_{ \pm} \in\left(A_{ \pm}\left(\tau_{s}\right)\right.$, $\left.A_{ \pm}\left(\tau_{s+1}\right)\right)$. By construction of $T_{\tau_{s}}^{\tau_{s+1}}\left(\right.$ see $\left.\S 2,\left(\mathrm{~b}_{0}\right)\right)$, for some $\tilde{\tau} \in\left(\tau_{s}, \tau_{s+1}\right), u_{0}=A_{0}(\tilde{\tau})$,

$$
u_{ \pm}=A_{ \pm}(\tilde{\tau})
$$

and $u_{0}$ is mapped to $u_{ \pm}$with probability $p_{ \pm}$,

$$
p_{ \pm}=p_{ \pm}(\tilde{\tau})=\frac{A_{ \pm}^{\prime}(\tilde{\tau})}{A_{0}^{\prime}(\tilde{\tau})} .
$$

Hence $p_{ \pm} /\left(d u_{ \pm} / d u_{0}\right)=1$. This equation coincides with (2.4) in case $(+++)$.

\section{CAMBRIDGE}


Case $(+ \pm), A_{+}^{\prime}<0$. In this case $T_{\tau_{s}}^{\tau_{s+1}}$ equals the identity on

$$
\left(A_{0}\left(\tau_{s+1}\right), C\right) \sqcup\left(0, A_{+}\left(\tau_{s+1}\right)\right) \sqcup\left(0, A_{-}\left(\tau_{s}\right)\right) .
$$

We only need to consider $\hat{\mathcal{T}}_{s}=\left.T_{\tau_{s}}^{\tau_{s+1}}\right|_{\left(A_{0}\left(\tau_{s}\right), A_{0}\left(\tau_{s+1}\right)\right) \sqcup\left(A_{+}\left(\tau_{s+1}\right), A_{+}\left(\tau_{s}\right)\right)}$

$$
\hat{\mathcal{T}}_{s}:\left(A_{0}\left(\tau_{s}\right), A_{0}\left(\tau_{s+1}\right)\right) \sqcup\left(A_{+}\left(\tau_{s+1}\right), A_{+}\left(\tau_{s}\right)\right) \rightarrow\left(A_{-}\left(\tau_{s}\right), A_{-}\left(\tau_{s+1}\right)\right) .
$$

Let $u_{-}$be an arbitrary point in $\left(A_{-}\left(\tau_{s}\right), A_{-}\left(\tau_{s+1}\right)\right)$. It has two preimages: $u_{0} \in$ $\left(A_{0}\left(\tau_{s}\right), A_{0}\left(\tau_{s+1}\right)\right)$ and $u_{+} \in\left(A_{+}\left(\tau_{s+1}\right), A_{+}\left(\tau_{s}\right)\right)$. By construction of $T_{\tau_{s}}^{\tau_{s+1}}$, for some $\tilde{\tau} \in\left(\tau_{s}, \tau_{s+1}\right)$,

$$
u_{-}=A_{-}(\tilde{\tau}), \quad u_{0}=A_{0}(\tilde{\tau}), \quad u_{+}=A_{+}(\tilde{\tau}),
$$

where $u_{0}$ and $u_{+}$are mapped to $u_{-}$with probability 1 . Hence

$$
\frac{1}{\left|d u_{-} / d u_{0}\right|}+\frac{1}{\left|d u_{-} / d u_{+}\right|}=\frac{A_{0}^{\prime}(\tilde{\tau})}{A_{-}^{\prime}(\tilde{\tau})}-\frac{A_{+}^{\prime}(\tilde{\tau})}{A_{-}^{\prime}(\tilde{\tau})}=1 .
$$

This equation coincides with (2.4) in the case $(+ \pm), A_{+}^{\prime}<0$.

\section{B. Appendix. Lower estimate for the functional $B$}

B.1. Proof of Lemma 6.1. Since $Q$ is positive, it is possible to assume that $Q \equiv 1$. Below we fix a point $x_{0} \in(0,1)$ and put $x_{1}=h\left(x_{0}\right)$. We have the equations

$$
\begin{gathered}
\|f\|^{2}=\int_{x_{0}}^{x_{1}} \sum_{-\infty}^{\infty} f^{2} \circ h^{k}(x)\left(h^{k}\right)^{\prime}(x) d x, \\
B(f)=\int_{x_{0}}^{x_{1}} \sum_{-\infty}^{\infty}\left(f \circ h^{k+1}(x)-f \circ h^{k}(x)\right)^{2}\left(h^{k}\right)^{\prime}(x) d x .
\end{gathered}
$$

Let $\operatorname{pr}_{H}: L_{2} \rightarrow H$ be the orthogonal projection. Then, for any $f \in H^{\perp}$,

$$
\operatorname{pr}_{H} f=\frac{\sum_{k \in \mathbb{Z}}\left(h^{k}\right)^{\prime} f \circ h^{k}}{\sum_{k \in \mathbb{Z}}\left(h^{k}\right)^{\prime}}=0 .
$$

For any $x \in\left[x_{0}, x_{1}\right]$ we put $\chi_{k}=\chi_{k}(x)=\left(h^{k}\right)^{\prime}(x)$. For definiteness we assume that in (iii) (§6.2) $0<\lambda_{1}<1<\lambda_{0}$. Then, by (i)-(iii), we have the following.

(1) $\chi_{k}>0$.

(2) $\lim _{k \rightarrow-\infty} \chi_{k} / \lambda_{0}^{k}$ and $\lim _{k \rightarrow+\infty} \chi_{k} / \lambda_{1}^{k}$ exist and are positive (i.e., $\chi_{k}(x), x \in\left[x_{0}, x_{1}\right]$ exponentially tends to zero as $k \rightarrow \pm \infty$ ).

(3) There exist $c_{1}, c_{2}>0$ such that

$$
c_{1} \chi_{k}(\xi) \leq \chi_{k}(x) \leq c_{2} \chi_{k}(\xi) \text { for any } k \in \mathbb{Z} \text { and any } x, \xi \in\left[x_{0}, x_{1}\right] .
$$

Conditions (1)-(3) and equation (B.2) give us the possibility to apply Lemmas B.1-B.2 (see Appendix B.2). For any $x \in\left[x_{0}, x_{1}\right]$ these lemmas estimate the integrand in (B.1) provided $f \in H^{\perp}$. Let $\gamma=\gamma(\chi(x))$ be defined by (B.3). By Lemmas B.1 and B.2, $\gamma(\chi(x))>\hat{\gamma}>0$. We have

$$
\begin{aligned}
B(f) & \geq \int_{x_{0}}^{x_{1}} \sum_{k=-\infty}^{+\infty} \gamma(\chi(x)) f^{2} \circ h^{k}(x)\left(h^{k}\right)^{\prime}(x) d x \\
& \geq \hat{\gamma} \int_{x_{0}}^{x_{1}} \sum_{k=-\infty}^{+\infty} f^{2} \circ h^{k}(x)\left(h^{k}\right)^{\prime}(x) d x=\hat{\gamma}\|f\|^{2} .
\end{aligned}
$$

Hence in (6.5) we can take $c=\hat{\gamma}$.

\section{CAMBridge}


B.2. Discrete version of Lemma 6.1. Consider the sequence of positive numbers $\ldots, \chi_{-1}, \chi_{0}, \chi_{1}, \ldots$ such that for some constants $0<\lambda_{1}<1<\lambda_{0}$ and $\alpha_{0}, \alpha_{1}>0$

$$
\lim _{n \rightarrow-\infty} \chi_{n} / \lambda_{0}^{n}=\alpha_{0}, \quad \lim _{n \rightarrow+\infty} \chi_{n} / \lambda_{1}^{n}=\alpha_{1} .
$$

Consider the real Hilbert space $l_{2}(\chi)$, formed by sequences $a=\left(\ldots, a_{0}, a_{1}, \ldots\right)$. The scalar product and the norm are determined by

$$
\langle a, b\rangle=\sum_{n \in \mathbb{Z}} \chi_{n} a_{n} b_{n}, \quad\|a\|=\sqrt{\langle a, a\rangle} .
$$

We put $1=(\ldots, 1,1, \ldots) \in l_{2}(\chi)$,

$$
\begin{gathered}
\mathcal{B}(a)=\sum_{n \in \mathbb{Z}} \chi_{n}\left(a_{n+1}-a_{n}\right)^{2}, \\
\gamma=\inf _{a \neq 0,\langle a, 1\rangle=0} \mathcal{B}(a)\|a\|^{-2}=\inf _{\|a\|=1,\langle a, l\rangle=0} \mathcal{B}(a) .
\end{gathered}
$$

LEMMA B.1. The constant $\gamma=\gamma(\chi)$ is positive.

LemMA B.2. Suppose that the sequences $\chi^{\prime}$ and $\chi^{\prime \prime}$ are such that for some positive constants $c_{1}<c_{2}$

$$
c_{1} \chi_{n}^{\prime \prime} \leq \chi_{n}^{\prime} \leq c_{2} \chi_{n}^{\prime \prime}, \quad n \in \mathbb{Z}
$$

Then

Proof of Lemma B.1. For any $N \in \mathbb{N}$ we put

$$
\frac{c_{1}}{c_{2}} \gamma\left(\chi^{\prime \prime}\right) \leq \gamma\left(\chi^{\prime}\right) \leq \frac{c_{2}}{c_{1}} \gamma\left(\chi^{\prime \prime}\right)
$$

$$
\begin{gathered}
\langle a, b\rangle_{N}=\sum_{n=-N}^{N} \chi_{n} a_{n} b_{n}, \quad\|a\|_{N}=\sqrt{\langle a, a\rangle_{N}}, \quad \mathcal{B}_{N}(a)=\sum_{n=-N}^{N-1} \chi_{n}\left(a_{n+1}-a_{n}\right)^{2}, \\
\langle a, b\rangle_{N}^{ \pm}=\sum_{ \pm n>N} \chi_{n} a_{n} b_{n}, \quad\|a\|_{N}^{ \pm}=\sqrt{\langle a, a\rangle_{N}^{ \pm}} \\
\mathcal{B}_{N}^{+}(a)=\sum_{n \geq N} \chi_{n}\left(a_{n+1}-a_{n}\right)^{2}, \quad \mathcal{B}_{N}^{-}(a)=\sum_{n<-N} \chi_{n}\left(a_{n+1}-a_{n}\right)^{2} .
\end{gathered}
$$

Then for all $a \in l_{2}(\chi)$ and $N \in \mathbb{N}$

$$
\|a\|^{2}=\|a\|_{N}^{2}+\left(\|a\|_{N}^{+}\right)^{2}+\left(\|a\|_{N}^{-}\right)^{2}, \quad \mathcal{B}(a)=B_{N}(a)+B_{N}^{+}(a)+B_{N}^{-}(a) .
$$

Let $a^{(1)}, a^{(2)}, \ldots \in l_{2}(\chi)$ be a sequence such that

$$
\left\langle a^{(j)}, l\right\rangle=0, \quad\left\|a^{(j)}\right\|=1 \quad \text { and } \quad \lim _{j \rightarrow \infty} \mathcal{B}\left(a^{(j)}\right)=\gamma .
$$

(1) Suppose that there exist $N, \beta>0$ such that $\left\|a^{(j)}\right\|_{N} \geq \beta$ for any $j \in \mathbb{N}$. Then

$$
\begin{aligned}
\left|\left\langle a^{(j)}, l\right\rangle_{N}\right| & =\left|\left\langle a^{(j)}, l\right\rangle_{N}^{+}+\left\langle a^{(j)}, l\right\rangle_{N}^{-}\right| \leq\left\|a^{(j)}\right\|_{N}^{+}\|1\|_{N}^{+}+\left\|a^{(j)}\right\|_{N}^{-}\|1\|_{N}^{-} \\
& \leq \sqrt{1-\beta^{2}}\left(\|1\|_{N}^{+}+\|1\|_{N}^{-}\right) .
\end{aligned}
$$

Obviously $\|1\|_{N}^{ \pm}$tend to zero as $N \rightarrow \infty$. Therefore (since we can assume that $N$ is sufficiently large)

$$
\left|\left\langle a^{(j)}, 1\right\rangle_{N}\right| \leq \frac{1}{2} \beta\|1\|_{N} .
$$

\section{CAMBrIDGE JDUNALS}


Then for any $j$

$$
\begin{aligned}
& \mathcal{B}\left(a^{(j)}\right) \geq \mathcal{B}_{N}\left(a^{(j)}\right) \geq \inf _{\|a\|_{N}=\beta,\left|\langle a, I\rangle_{N}\right| \leq(1 / 2) \beta\|1\|_{N}} \mathcal{B}_{N}(a) \\
& =\beta^{2} \inf _{\|a\|_{N}=1,\left|\langle a, I\rangle_{N}\right| \leq(1 / 2)\|1\|_{N}} \mathcal{B}_{N}(a) .
\end{aligned}
$$

For any $N>0$ the last infimum is positive because it is, in fact, a minimum of a strictly positive continuous function of $2 N+1$ variables on a compact set.

(2) Suppose that there exists a subsequence $j_{s}$ such that $\left\|a^{\left(j_{s}\right)}\right\|_{N} \rightarrow 0$ as $s \rightarrow \infty$ for any $N>0$. We assume that $N$ is so large that

$$
\left|\chi_{-n} / \lambda_{0}^{-n}-\alpha_{0}\right|<\varepsilon_{1} \alpha_{0}, \quad\left|\chi_{n} / \lambda_{1}^{n}-\alpha_{1}\right|<\varepsilon_{1} \alpha_{1} \text { for any } n \geq N,
$$

where $\varepsilon_{1}>0$ can be chosen arbitrarily small. Consider $a=a^{\left(j_{s}\right)}$ such that

$$
\begin{aligned}
& \|a\|_{N+1}<\varepsilon_{2}, \\
& \mathcal{B}(a)<\gamma+\varepsilon_{3} .
\end{aligned}
$$

Then

$$
\left(\|a\|_{N+1}^{+}\right)^{2}+\left(\|a\|_{N+1}^{-}\right)^{2}>1-\varepsilon_{2}^{2} .
$$

One of the two terms in the left-hand side of this inequality is greater than $\left(1-\varepsilon_{2}^{2}\right) / 2$. For definiteness, we assume that

$$
\left(\|a\|_{N+1}^{+}\right)^{2}>\left(1-\varepsilon_{2}^{2}\right) / 2 .
$$

For any $a \in l_{2}(\chi)$ we define $\vec{a} \in l_{2}(\chi)$ such that $\vec{a}_{n}=a_{n+1}$ for all $n \in \mathbb{Z}$. Then

$$
B_{N}^{+}(a)=\left(\|a\|_{N}^{+}\right)^{2}+\left(\|\vec{a}\|_{N}^{+}\right)^{2}-2\langle a, \vec{a}\rangle_{N}^{+} \geq\left(\|a\|_{N}^{+}-\|\vec{a}\|_{N}^{+}\right)^{2} .
$$

Proposition B.1. Inequalities (B.5)-(B.6) imply

$$
\left(\|a\|_{N}^{+}\right)^{2}=\lambda_{1}\left(\|\vec{a}\|_{N}^{+}\right)^{2}+\mathcal{R},
$$

where

$$
|\mathcal{R}| \leq \varepsilon_{2}^{2}+\frac{2 \varepsilon_{1}}{1-\varepsilon_{1}}\left(\|a\|_{N+1}^{+}\right)^{2} .
$$

Proof of Proposition B.1. By definition

$$
\begin{aligned}
& \left(\|a\|_{N}^{+}\right)^{2}=\sum_{n=N+1}^{\infty} \chi_{n} a_{n}^{2}=\lambda_{1}\left(\|\vec{a}\|_{N}^{+}\right)^{2}+\mathcal{R}, \\
& \mathcal{R}=\chi_{N+1} a_{N+1}^{2}+\sum_{n=N+2}^{\infty}\left(\chi_{n}-\lambda_{1} \chi_{n-1}\right) a_{n}^{2} .
\end{aligned}
$$

Note that by (B.5)

$$
\left|\chi_{n}-\lambda_{1} \chi_{n-1}\right|=\lambda_{1}^{n}\left|\left(\chi_{n} \lambda_{1}^{-n}-\alpha_{1}\right)-\left(\chi_{n-1} \lambda_{1}^{1-n}-\alpha_{1}\right)\right| \leq 2 \varepsilon_{1} \lambda_{1}^{n} \alpha_{1} .
$$

Therefore again by (B.5)

$$
\begin{aligned}
\sum_{n=N+2}^{\infty}\left(\chi_{n}-\lambda_{1} \chi_{n-1}\right) a_{n}^{2} & \leq \sum_{n=N+2}^{\infty} 2 \varepsilon_{1} \alpha_{1} \lambda_{1}^{n} a_{n}^{2} \\
& \leq \sum_{n=N+2}^{\infty} \frac{2 \varepsilon_{1} \chi_{n} a_{n}^{2}}{1-\varepsilon_{1}}=\frac{2 \varepsilon_{1}}{1-\varepsilon_{1}}\left(\|a\|_{N+1}^{+}\right)^{2} .
\end{aligned}
$$

Inequalities (B.6) and (B.11) imply estimate (B.10). 
Proposition B.1 and estimates (B.8) and (B.9) imply that

$$
\begin{aligned}
B_{N}^{+}(a) & \geq\left(\|a\|_{N}^{+}-\|\vec{a}\|_{N}^{+}\right)^{2}=\frac{\left(\left(\|a\|_{N}^{+}\right)^{2}-\left(\|\vec{a}\|_{N}^{+}\right)^{2}\right)^{2}}{\left(\|a\|_{N}^{+}+\|\vec{a}\|_{N}^{+}\right)^{2}} \\
& \geq \frac{1}{4}\left(\left(\frac{1}{\lambda_{1}}-1\right)\left(\|a\|_{N}^{+}\right)^{2}-\frac{|R|}{\lambda_{1}}\right)^{2} \\
& \geq \frac{1}{4}\left(\left(\frac{1}{\lambda_{1}}-1-\frac{2 \varepsilon_{1}}{\lambda_{1}\left(1-\varepsilon_{1}\right)}\right) \frac{1-\varepsilon_{2}^{2}}{2}-\varepsilon_{2}^{2}\right)^{2} .
\end{aligned}
$$

It follows from (B.7) that the last expression is smaller than $\gamma+\varepsilon_{3}$. If $\varepsilon_{1}, \varepsilon_{2}$, and $\varepsilon_{3}$ are sufficiently small, this means that $\gamma>\left(1-\lambda_{1}\right)^{2} / 16 \lambda_{1}^{2}>0$.

The case, when instead of (B.8) we have $\left(\|a\|_{N+1}^{-}\right)^{2}>\left(1-\varepsilon_{2}^{2}\right) / 2$, is analogous.

Proof of Lemma B.2. The vector spaces $l_{2}\left(\chi^{\prime}\right)$ and $l_{2}\left(\chi^{\prime \prime}\right)$ coincide. Let $\|\cdot\|^{\prime}$ and $\|\cdot\|^{\prime \prime}$ be the corresponding norms. Analogously we define $\mathcal{B}^{\prime}$ and $\mathcal{B}^{\prime \prime}$. Now estimates (B.4) follow from the inequalities

$$
\alpha_{1}\|a\|^{\prime \prime} \leq\|a\|^{\prime} \leq \alpha_{2}\|a\|^{\prime \prime}, \quad \alpha_{1} \mathcal{B}^{\prime \prime}(a) \leq \mathcal{B}^{\prime}(a) \leq \alpha_{2} \mathcal{B}^{\prime \prime}(a) .
$$

\section{Appendix. Proof of Lemma 5.1}

Take any sequence $\left\{a_{n}\right\}, n \in \mathbb{Z}$ such that the following hold.

(i) $a_{n}+a_{-n}=1$ for all $n \in \mathbb{Z}$.

(ii) $a_{n}<a_{n+1}$ for any $n \in \mathbb{Z}$.

(iii) The sequence $a_{n}-a_{n+1}, n=0,1, \ldots$ is decreasing.

(iv) $\lim _{n \rightarrow-\infty} a_{n}=0, \lim _{n \rightarrow+\infty} a_{n}=1$.

(v) $\quad \sum_{n=-\infty}^{+\infty}\left(a_{2 n}-a_{2 n-1}\right)=1 / 2$.

(vi) $\left(a_{n}-a_{n-1}\right) /\left(a_{n-1}-a_{n-2}\right)=e^{\lambda}(1+O(1 / n))$ for $n \rightarrow-\infty$.

For example, we can take

$$
a_{n}=\frac{1}{\sinh (\lambda / 2)} \sum_{k=-\infty}^{n} e^{-\lambda|k-1 / 2|}
$$

Consider the function $h_{0}: \mathbb{R} \rightarrow(0,1)$ such that $h_{0}(n)=a_{n}$ for $n \in \mathbb{Z}$ and $h_{0}$ is linear on any interval $(n, n+1)$. Then $h_{0}$ satisfies conditions (2) and (3) of the lemma.

Instead of condition (4), $h_{0}$ satisfies the following condition:

$$
h_{0}^{\prime}(y) \geq h_{0}^{\prime}(y+1) \quad \text { for any } y>-1, y \notin \mathbb{Z} .
$$

The function $\chi(y)=\sum_{n=-\infty}^{+\infty}\left(h_{0}(2 n+y)-h_{0}(2 n-1+y)\right)$ is 2-periodic in $y \in \mathbb{R}$, piecewise linear, and smooth outside $\mathbb{Z}$. By $(v) \chi(0)=1 / 2$. Since $\chi(0)+\chi(1)=1$, we have $\chi(1)=1 / 2$. Therefore $\chi \equiv 1 / 2$ and condition (5) also holds.

Condition (vi) implies that

$$
h_{0}^{\prime}(y) / h_{0}^{\prime}(y-1)=e^{\lambda}(1+O(1 / y)) \text { for } y \rightarrow-\infty, y \notin \mathbb{Z} .
$$

To satisfy conditions (1), (4), and (6), we perform a smoothing of $h_{0}$. To this end we consider a smooth even function $\omega: \mathbb{R} \rightarrow[0,+\infty)$ such that

$$
\omega(\xi)=0 \text { for }|\xi| \geq 1 \text { and } \int_{-\infty}^{+\infty} \omega(\xi) d \xi=1
$$

\section{CAMBRIDGE}


We put

$$
h(y)=\int_{-\infty}^{+\infty} h_{0}(\xi) \omega(y-\xi) d \xi \equiv \int_{-\infty}^{+\infty} h_{0}(y-\xi) \omega(\xi) d \xi .
$$

The function $h$ satisfies conditions (1)-(3). For any $y \geq 0$, (C.1) implies that

$$
h^{\prime}(y)-h^{\prime}(y+1)=\int_{-1}^{1}\left(h_{0}^{\prime}(y-\xi)-h_{0}^{\prime}(y+1-\xi)\right) \omega(\xi) d \xi \geq 0,
$$

i.e., condition (4) holds. Condition (5) follows from the equation

$$
\begin{aligned}
& \sum_{n=-\infty}^{+\infty}(h(2 n+y)-h(2 n-1+y)) \\
& \quad=\int_{-\infty}^{+\infty} \sum_{n=-\infty}^{+\infty}\left(h_{0}(2 n+y-\xi)-h_{0}(2 n-1+y-\xi)\right) \omega(\xi) d \xi \\
& =\int_{-\infty}^{+\infty} \frac{1}{2} \omega(\xi) d \xi=\frac{1}{2} .
\end{aligned}
$$

By using (C.2) we obtain

$$
\begin{aligned}
h^{\prime}(y) & =\int_{-1}^{1} h_{0}^{\prime}(y-\xi) \omega(\xi) d \xi=\int_{-1}^{1} e^{\lambda}(1+o(1 / y)) h_{0}^{\prime}(-1+y-\xi) \omega(\xi) d \xi \\
& =e^{\lambda} h^{\prime}(y-1)(1+O(1 / y)) .
\end{aligned}
$$

This equation implies condition (6).

\section{Appendix. Proof of Lemma 7.1}

Without loss of generality we assume that $a=0$ and $b=1$. For any $\alpha<\beta$ we define the set

$$
U_{\alpha, \beta}=\{x \in[0,1]: \alpha<g(x)<\beta\} .
$$

The lemma will be proven if for any $\alpha<\beta$ we show that the inequality $\left|U_{\alpha, \beta}\right|>0$ (where $|\cdot|$ is the Lebesgue measure) implies the estimate $\left|U_{\alpha, \beta}\right|>1 / 2$. Indeed, if $g$ does not coincide almost everywhere with a constant, there exist $\alpha<\beta<\alpha^{\prime}<\beta^{\prime}$ such that $\left|U_{\alpha, \beta}\right|>0$ and $\left|U_{\alpha^{\prime}, \beta^{\prime}}\right|>0$. Then we obtain

$$
\left|U_{\alpha, \beta}\right|+\left|U_{\alpha^{\prime}, \beta^{\prime}}\right|>1=|[0,1]| .
$$

Below we fix $\alpha$ and $\beta$ and, for brevity, use the notation $U$ instead of $U_{\alpha, \beta}$.

Suppose that $|U|>0$. Then there exists a density point $x_{0} \in(0,1)$. This means that for any $\varepsilon>0$ there exists an interval $I_{\varepsilon} \subset[0,1], x_{0} \in I_{\varepsilon}$ such that

$$
\frac{\left|U \cap I_{\varepsilon}\right|}{\left|I_{\varepsilon}\right|}>1-\varepsilon
$$

We put $h_{0}(1)=A$ and $h_{1}(0)=B$. Since $\lambda_{0}+\lambda_{1}>1$, we have $A>B$. Now we consider the sequence of intervals

$$
K_{0}, K_{1}, \ldots, K_{N} \subset[0,1],
$$

constructed inductively as follows.

\section{CAMBRIDGE}


(0) $K_{0}=I_{\varepsilon}$

(1) If $K_{n} \subset(0, A)$, we put $K_{n+1}=h_{0}^{-1}\left(K_{n}\right)$.

(2) If $K_{n} \not \subset(0, A)$ but $K_{n} \subset(B, 1)$, we put $K_{n+1}=h_{1}^{-1}\left(K_{n}\right)$.

(3) We finish if $K_{n} \not \subset(0, A)$ and $K_{n} \not \subset(B, 1)$ (i.e., $\left.(B, A) \subset K_{n}\right)$.

Since $\lambda_{0}, \lambda_{1}<1$, the sequence $\left|K_{0}\right|,\left|K_{1}\right|, \ldots$ grows exponentially. Therefore the sequence stops at some finite step $n=N$. Note also that because of the condition $g=g \circ h_{0}=g \circ h_{1}$ we have

$$
\frac{\left|U \cap K_{n}\right|}{\left|K_{n}\right|}>1-\varepsilon \text { for all } n=0, \ldots, N .
$$

The estimate $\left|K_{N} \backslash U\right|<\varepsilon\left|K_{N}\right| \leq \varepsilon$ and the inclusion $(B, A) \subset K_{N}$ imply that $|(B, A) \backslash U| \leq \varepsilon$. Therefore

$$
\frac{|U \cap(B, A)|}{|(B, A)|}>1-\frac{\varepsilon}{A-B} .
$$

At least one of the inequalities $|(0, A)|>1 / 2,|(B, 1)|>1 / 2$ holds. For definiteness let it be the second one. Applying the map $h_{0}^{-1}$, we have

$$
\frac{|U \cap(C, 1)|}{|(C, 1)|}>1-\frac{\varepsilon}{A-B}, \quad C=h_{0}^{-1}(B) .
$$

Now we consider the interval

$$
J=h_{1}^{-s}((C, 1))=\left(h_{1}^{-s}(C), 1\right)
$$

with minimal $s \in\{0,1,2, \ldots\}$ such that $B \in J$. Then

$$
|J|>|(B, 1)|>1 / 2 .
$$

The estimate $|U \cap J| /|J|>1-\varepsilon /(A-B)$ implies that

$$
|U| \geq|U \cap J|>|J|\left(1-\frac{\varepsilon}{A-B}\right) .
$$

By (D.1) the last expression is greater than $1 / 2$ for sufficiently small $\varepsilon$. Hence we obtain the required inequality $|U|>1 / 2$.

\section{REFERENCES}

[1] V. I. Arnold, V. V. Kozlov and A. I. Neishtadt. Mathematical Aspects of Classical and Celestial Mechanics. Springer, Berlin, 2006.

[2] J. R. Cary, D. F. Escande and J. L. Tennyson. Adiabatic invariant change due to separatrix crossing. Phys. Rev. A 34(5) (1986), 4256-4275.

[3] U. Krengel. Ergodic Theorems. Walter de Gruyter, Berlin, 1985.

[4] A. I. Neishtadt. Passage through a separatrix in a resonance problem with a slowly-varying parameter. Prikl. Mat. Mekh. 39(4) (1975), 621-632 (Engl. transl. J. Appl. Math. Mech. 39(4) (1975), 594-605).

[5] A. I. Neishtadt. Change of an adiabatic invariant at a separatrix. Fiz. Plazmy 12(8) (1986), 992-1000 (Engl. transl. Sov. J. Plasma Phys. 12 (1986), 568-573).

[6] A. V. Timofeev. On the constancy of an adiabatic invariant when the nature of the motion changes. Zh. Ehksper. Teor. Fiz. 75(4) (1978), 1303-1308 (Engl. transl. Sov. Phys., JETP 48 (1978), 656-659).

[7] A. M. Vershik. Multivalued mappings with invariant measure (polymorphisms) and Markov operators. Zap. Nauchn. Semin. LOMI 72 (1977), 26-61 (Engl. transl. J. Sov. Math. 23 (1983), 2243-2266).

[8] A. M. Vershik. Polymorphisms, Markov processes, and quasi-similarity. Discrete Contin. Dyn. Syst. 13(5) (2005), 1305-1324. 